Southern Illinois University Edwardsville SPARK

SIUE Faculty Research, Scholarship, and Creative Activity

2015

\title{
Kravchuk Polynomials and Induced/Reduced Operators on Clifford Algebras
}

G. Stacey Staples

Southern Illinois University Edwardsville, sstaple@siue.edu

Follow this and additional works at: http://spark.siue.edu/siue_fac

Part of the Algebra Commons, and the Discrete Mathematics and Combinatorics Commons

\section{Recommended Citation}

G.S. Staples, Kravchuk polynomials and induced/reduced operators on Clifford algebras, Complex Analysis and Operator Theory, 9 (2015), 445 - 478. http://dx.doi.org/10.1007/s11785-014-0377-z

This Article is brought to you for free and open access by SPARK. It has been accepted for inclusion in SIUE Faculty Research, Scholarship, and Creative Activity by an authorized administrator of SPARK. For more information, please contact gpark@siue.edu. 


\section{Cover Page Footnote}

The definitive version of this article was published by Springer in Complex Analysis and Operator Theory. The final publication is available at Springer via http://dx.doi.org/10.1007/s11785-014-0377-z . 


\title{
Kravchuk Polynomials and Induced/Reduced Operators on Clifford Algebras
}

\author{
G. Stacey Staples*
}

\begin{abstract}
Kravchuk polynomials arise as orthogonal polynomials with respect to the binomial distribution and have numerous applications in harmonic analysis, statistics, coding theory, and quantum probability. The relationship between Kravchuk polynomials and Clifford algebras is multifaceted. In this paper, Kravchuk polynomials are discovered as traces of conjugation operators in Clifford algebras, and appear in Clifford Berezin integrals of Clifford polynomials. Regarding Kravchuk matrices as linear operators on a vector space $V$, the action induced on the Clifford algebra over $V$ is equivalent to blade conjugation, i.e., reflections across sets of orthogonal hyperplanes. Such operators also have a natural interpretation in terms of raising and lowering operators on the algebra. On the other hand, beginning with particular linear operators on the Clifford algebra $\mathcal{C} \ell_{Q}(V)$, one obtains Kravchuk matrices as operators on the paravector space $V_{*}$ through a process of operator grade-reduction. Symmetric Kravchuk matrices are recovered as representations of grade-reductions of maps induced by negative-definite quadratic forms on $V$.
\end{abstract}

AMS Subj. Classifications: 15A66, 47C05, 81R05, 60B99

Keywords: Operator calculus, Kravchuk polynomials, Clifford algebras, quantum probability

\section{Introduction and Notational Preliminaries}

Applications of Kravchuk polynomials are widespread. As orthogonal polynomials, they appear in the classic work by Szëgo [27]. They have been studied from the point of view of harmonic analysis and special functions, as in work of Dunkl $[7,8]$. Kravchuk polynomials may be viewed as the discrete version of Hermite polynomials [1].

In combinatorics and coding theory, Kravchuk polynomials are essential in MacWilliams' theorem on weight enumerators [17], [19]. They also provide fundamental examples in association schemes [4], [5], [6].

*Department of Mathematics and Statistics, Southern Illinois University Edwardsville, Edwardsville, IL 62026-1653,USA, Email: sstaple@siue.edu 
In the context of the classical symmetric random walk, Kravchuk polynomials are elementary symmetric functions in variables taking values \pm 1 . The generating function is a martingale in the parameter $n$ [14].

In quantum theory, Kravchuk matrices interpreted as operators give rise to two new interpretations in the context of both classical and quantum random walks [12]. The latter interpretation underlies the basis of quantum computing.

In Koornwinder's work, Kravchuk polynomials are interpreted as spherical functions as well as intertwining functions on wreath products of symmetric groups. Moreover, Kravchuk polynomials can be interpreted as matrix elements for representations of $S U(2)$ [16].

Several notions of induced and reduced operators will be introduced throughout the paper. Generally, the term induced operator will refer to an operator on a Clifford algebra $\mathcal{C} \ell_{Q}(V)$ obtained from an operator on the underlying vector space $V$ spanned by the algebra's generators. The term reduced operator will generally refer to an operator on the paravector space $V_{*}:=\mathbb{R} \oplus V$ obtained from an operator on the Clifford algebra. Finally, a deduced operator will be an operator on $V$ obtained by restricting an operator on the full algebra, provided the operator on $V$ also induces the operator on the full algebra.

Given an algebraic element $\mathfrak{u}$ known as a blade, the action $\mathbf{x} \mapsto \mathfrak{u x u}^{-1}$ is referred to as blade conjugation. As will be seen, Kravchuk polynomials arise naturally from the characteristic polynomials of blade conjugation operators. These polynomials are also recovered from Berezin integrals of Clifford polynomials.

Further, under conditions specified later, the $n^{\text {th }}$ Kravchuk matrix can be naturally interpreted as a blade conjugation operator on an $(n+1)$-dimensional vector space. Symmetric Kravchuk matrices have a natural interpretation as linear operators on paravector spaces and are obtained as grade reductions of form-induced operators on Clifford algebras.

\subsection{Kravchuk polynomials and Kravchuk matrices}

The three-term recurrence relation for the Kravchuk polynomials of order $n$ is as follows. Define $K_{0}(x ; n):=1$ and $K_{1}(x ; n):=x$. For $\ell \geq 2$, the $\ell^{\text {th }}$ Kravchuk polynomial is given by

$$
K_{\ell}(x ; n):=x K_{\ell-1}(x ; n)+(\ell-1)(n-\ell+2) K_{\ell-2}(x ; n) .
$$

An explicit formula for the $n^{\text {th }}$ Kravchuk polynomial is given by

$$
K_{\ell}(x, n):=\sum_{i=0}^{n}(-1)^{i}\left(\begin{array}{c}
x \\
i
\end{array}\right)\left(\begin{array}{c}
n-x \\
\ell-i
\end{array}\right) .
$$

Consider a Bernoulli random walk starting at the origin, jumping to the left with probability $q$ and to the right with probability $p$, where $p+q=1$ and $p q \neq 0$. After $n$ steps, the position is $x=n-2 j$, where $j$ denotes the number 
of jumps to the left. A generating function for Kravchuk polynomials is then given by

$$
G(v)=(1+v)^{(n+x) / 2}(1-v)^{(n-x) / 2}=\sum_{\ell=0}^{n} \frac{v^{\ell}}{\ell !} K_{\ell}(x, n) .
$$

As functions of $j$, the generating function can be written

$$
G(v)=(1+v)^{n-j}(1-v)^{j}=\sum_{\ell=0}^{n} \frac{v^{\ell}}{\ell !} K_{\ell}(j, n) .
$$

Let $\left\{\varepsilon_{i}: 1 \leq i \leq n+1\right\}$ denote the standard basis for $\mathbb{R}^{n+1}$. As defined in the work of Feinsilver and Fitzgerald [9], the $n^{\text {th }}$ Kravchuk matrix, $\mathfrak{K}_{n}$, is the $(n+1) \times(n+1)$ matrix defined via the Kravchuk polynomial generating function according to

$$
(1+x)^{n-j}(1-x)^{j}=\sum_{i=0}^{n} x^{i}\left\langle i\left|\mathfrak{K}_{n}\right| j\right\rangle,
$$

with $\left\langle i\left|\mathfrak{K}_{n}\right| j\right\rangle=K_{i}(j ; n)$, the $i^{\text {th }}$ Kravchuk polynomial evaluated at $j$.

Beginning with the Hadamard matrix:

$$
\mathfrak{K}_{1}=\left(\begin{array}{cc}
1 & 1 \\
1 & -1
\end{array}\right)
$$

the next few Kravchuk matrices are seen to be

$$
\begin{gathered}
\mathfrak{K}_{2}=\left(\begin{array}{ccc}
1 & 1 & 1 \\
2 & 0 & -2 \\
1 & -1 & 1
\end{array}\right), \quad \mathfrak{K}_{3}=\left(\begin{array}{cccc}
1 & 1 & 1 & 1 \\
3 & 1 & -1 & -3 \\
3 & -1 & -1 & 3 \\
1 & -1 & 1 & -1
\end{array}\right), \\
\mathfrak{K}_{4}=\left(\begin{array}{ccccc}
1 & 1 & 1 & 1 & 1 \\
4 & 2 & 0 & -2 & -4 \\
6 & 0 & -2 & 0 & 6 \\
4 & -2 & 0 & 2 & -4 \\
1 & -1 & 1 & -1 & 1
\end{array}\right) .
\end{gathered}
$$

The symmetric Kravchuk matrices $\mathfrak{K}_{n}{ }^{\mathrm{sym}}$ are defined by $\mathfrak{K}_{n}{ }^{\mathrm{sym}}:=\mathfrak{K}_{n} B$, where $B:=\left(b_{i i}\right)$ is the $(n+1) \times(n+1)$ diagonal matrix of binomial coefficients; i.e., $b_{i i}=\left(\begin{array}{c}n \\ i\end{array}\right)$ for $i=0, \ldots, n$. The first few symmetric Kravchuk matrices are

$$
\mathfrak{K}_{2}{ }^{\mathrm{sym}}:=\left(\begin{array}{ccc}
1 & 2 & 1 \\
2 & 0 & -2 \\
1 & -2 & 1
\end{array}\right), \quad \mathfrak{K}_{3}{ }^{\mathrm{sym}}:=\left(\begin{array}{cccc}
1 & 3 & 3 & 1 \\
3 & 3 & -3 & -3 \\
3 & -3 & -3 & 3 \\
1 & -3 & 3 & -1
\end{array}\right),
$$




$$
\mathfrak{K}_{4}{ }^{\text {sym }}:=\left(\begin{array}{ccccc}
1 & 4 & 6 & 4 & 1 \\
4 & 8 & 0 & -4 & -4 \\
6 & 0 & -12 & 0 & 6 \\
4 & -8 & 0 & 8 & -4 \\
1 & -4 & 6 & -4 & 1
\end{array}\right) .
$$

For convenience, a summary of the notation used throughout this paper appears in Table 1.

\begin{tabular}{|c|c|}
\hline Notation & Meaning \\
\hline$K_{\ell}(x ; n)$ & $\ell^{\text {th }}$ Kravchuk polynomial of order $n$ \\
$\mathfrak{K}_{n}$ & $n^{\text {th }}$ Kravchuk matrix \\
$\mathfrak{K}_{n}^{\text {sym }}$ & $n^{\text {th }}$ symmetric Kravchuk matrix \\
$\mathcal{S}_{n}$ & Symmetric group of order $n !$ \\
$\mathcal{K}_{n}$ & $n^{\text {th }}$ normalized Kravchuk matrix \\
$\omega$ & Unit pseudoscalar \\
$\mathbf{u}_{i}$ & Vector: lowercase, bold, single index. \\
$\varepsilon_{i}$ & Canonical basis for paravectors $V_{*}:=\mathbb{R} \oplus V, 0 \leq i \leq|V|$. \\
$\mathfrak{u}$ & Blades; mathfrak $\mathfrak{u}=\mathbf{u}_{1} \wedge \cdots \wedge \mathbf{u}_{k}$ \\
$\varphi_{\mathfrak{u}}$ & Blade conjugation operator on $\mathbf{x} \mapsto \mathfrak{u x} \mathfrak{u}^{-1}$ \\
$\Phi_{\mathfrak{u}}$ & Restriction of blade conjugation operator $\varphi_{\mathfrak{u}}$ to $V$ \\
$\varphi_{\mathfrak{u}}(\ell)$ & Restriction of blade conjugation operator $\varphi_{\mathfrak{u}}$ to \\
& grade- $\ell$ subspace of $\mathcal{C} \ell_{Q}(V)$ \\
$\check{A}$ & Matrix representation of linear operator $A$ \\
$\psi_{\mathfrak{u}}$ & Generalized blade conjugation operator $\mathbf{x} \mapsto \mathfrak{u x} \tilde{\mathfrak{u}}$ \\
$\Psi_{\mathfrak{u}}$ & Restriction of generalized blade conjugation $\psi_{\mathfrak{u}}$ to $V$ \\
$\mathbf{u}_{I}$ & Multi-index notation for basis blades. Ordered product. \\
& und $:=\prod_{\ell \in I} \mathbf{u}_{\ell}=\mathbf{u}_{I_{1}} \wedge \cdots \wedge \mathbf{u}_{I I I} \cdot$ \\
$\sharp \mathfrak{u}$ & Grade of blade $\mathfrak{u} ;$ i.e., $\mathfrak{u}:=\mathbf{u}_{1} \wedge \cdots \wedge \mathbf{u}_{\sharp \mathfrak{u}}$. \\
$\langle\xi\rangle_{\ell}$ & Grade- $\ell$ part of $\xi \in \mathcal{C} \ell_{Q}(V)$ \\
$\pi_{\ell}$ & Canonical grade- $\ell$ projection operator: $\pi_{\ell}(x):=\langle x\rangle_{\ell}$. \\
$\mathbb{R}^{*}$ & Invertible real numbers, $\mathbb{R}^{*}:=\mathbb{R} \backslash\{0\}$ \\
$\mathfrak{x} \mid \mathfrak{u}$ & $\mathfrak{u}=\mathfrak{x} \mathfrak{v}$ for some blade $\mathfrak{v}$ with $\sharp \mathfrak{u}=\sharp \mathfrak{x}+\sharp \mathfrak{v}$. \\
$\langle\mathbf{1}|$ & Row vector of all ones. \\
\hline
\end{tabular}

Table 1: Summary of Notation

\subsection{Clifford Algebras}

Let $V$ be an $n$-dimensional vector space over $\mathbb{R}$ equipped with a nondegenerate quadratic form $Q$. Associate with $Q$ the symmetric bilinear form

$$
\langle\mathbf{x}, \mathbf{y}\rangle_{Q}=\frac{1}{2}[Q(\mathbf{x}+\mathbf{y})-Q(\mathbf{x})-Q(\mathbf{y})],
$$


and extend to simple $k$-vectors in $\bigwedge^{k} V$ by

$$
\left\langle\mathbf{x}_{1} \wedge \mathbf{x}_{2} \wedge \cdots \wedge \mathbf{x}_{k}, \mathbf{y}_{1} \wedge \mathbf{y}_{2} \wedge \cdots \wedge \mathbf{y}_{k}\right\rangle_{Q}=\operatorname{det}\left\langle\mathbf{x}_{i}, \mathbf{y}_{j}\right\rangle_{Q}
$$

This inner product extends linearly to all of $\bigwedge^{k} V$ and by orthogonality to $\bigwedge V$.

The Clifford algebra $\mathcal{C} \ell_{Q}(V)$ is the real algebra obtained from associative linear extension of the Clifford vector product

$$
\mathbf{x} \mathbf{y}:=\langle\mathbf{x}, \mathbf{y}\rangle_{Q}+\mathbf{x} \wedge \mathbf{y}, \forall \mathbf{x}, \mathbf{y} \in V .
$$
by

Given a nondegenerate quadratic form $Q$, the mapping $\|\cdot\|_{Q}: V \rightarrow \mathbb{R}$ defined

$$
\|\mathbf{x}\|_{Q}=\left|\langle\mathbf{x}, \mathbf{x}\rangle_{Q}\right|^{1 / 2}, \quad(\mathbf{x} \in V)
$$

is readily seen to be a seminorm, referred to henceforth as the $Q$-seminorm on $V$.

A vector $\mathbf{x}$ is said to be anisotropic if $\|\mathbf{x}\|_{Q} \neq 0$. A set $S$ of $Q$-orthogonal vectors is said to be $Q$-orthonormal if $\|\mathbf{x}\|_{Q}=1$ for all $\mathbf{x} \in S$.

Note that since $Q$ is nondegenerate, all vectors of a $Q$-orthogonal basis for $V$ must be anisotropic. Given a collection of $Q$-orthogonal vectors $\left\{\mathbf{x}_{i}\right\}$, a $Q$ orthonormal basis $\left\{\mathbf{u}_{i}: 1 \leq i \leq n\right\}$ for $V$ is obtained by defining

$$
\mathbf{u}_{i}:=\frac{\mathbf{x}_{i}}{\left\|\mathbf{x}_{i}\right\|_{Q}}
$$

for each $i=1, \ldots, n$. In particular, for each $i=1, \ldots, n$,

$$
\mathbf{u}_{i}^{2}=\left\langle\mathbf{u}_{i}, \mathbf{u}_{i}\right\rangle_{Q}=\frac{\left\langle\mathbf{x}_{i}, \mathbf{x}_{i}\right\rangle_{Q}}{\left|\left\langle\mathbf{x}_{i}, \mathbf{x}_{i}\right\rangle_{Q}\right|}= \pm 1 .
$$

These vectors then generate the Clifford algebra $\mathcal{C} \ell_{Q}(V)$.

Generally speaking, the exterior product of $k$ linearly independent vectors is called a $k$-blade or blade of grade $k$. When the vectors are $Q$-orthogonal, one sees from (1.8) that the Clifford product coincides with the exterior product.

Given an arbitrary $Q$-orthogonal basis $\left\{\mathbf{v}_{i}: 1 \leq i \leq n\right\}$ for $V$, multi-index notation for canonical basis blades is adopted in the following manner. Denote the $n$-set $\{1, \ldots, n\}$ by $[n]$, and denote the associated power set by $2^{[n]}$. The ordered product of basis vectors (i.e., algebra generators) is then conveniently denoted by

$$
\prod_{i \in I} \mathbf{v}_{i}=\mathbf{v}_{I}
$$

for any subset $I \subseteq[n]$, also denoted $I \in 2^{[n]}$.

These products of generators are referred to as basis blades for the algebra. The grade of a basis blade is defined to be the cardinality of its multi-index. An arbitrary element $u \in \mathcal{C} \ell_{Q}(V)$ has a canonical basis blade decomposition of the form

$$
u=\sum_{I \subseteq[n]} u_{I} \mathbf{v}_{I},
$$


where $u_{I} \in \mathbb{R}$ for each multi-index $I$. The grade- $k$ part of $u \in \mathcal{C} \ell_{Q}(V)$ is then naturally defined by $\langle u\rangle_{k}:=\sum_{|I|=k} u_{I} \mathbf{v}_{I}$. It is now evident that $\mathcal{C} \ell_{Q}(V)$ has a canonical vector space decomposition of the form

$$
\mathcal{C} \ell_{Q}(V)=\bigoplus_{k=0}^{n}\left\langle\mathcal{C} \ell_{Q}(V)\right\rangle_{k}
$$

An arbitrary element $u \in \mathcal{C} \ell_{Q}(V)$ is said to be homogeneous of grade $k$ if $\langle u\rangle_{k} \neq 0$ and $\langle u\rangle_{\ell}=0$ for all $\ell \neq k$. As the degree of a polynomial refers to the maximal exponent appearing in terms of the polynomial, an arbitrary multivector $u \in \mathcal{C} \ell_{Q}(V)$ is said to be heterogeneous of grade $k$ if $\langle u\rangle_{k} \neq 0$ and $\langle u\rangle_{\ell}=0$ for $\ell>k$.

Definition 1.1. Given an arbitrary blade $\mathfrak{u} \in \mathcal{C} \ell_{Q}(V)$, a collection of vectors $\mathfrak{U}=\left\{\mathbf{u}_{1}, \ldots, \mathbf{u}_{\sharp \mathfrak{u}}\right\}$ is said to constitute an exterior factorization of $\mathfrak{u}$ if

$$
\mathfrak{u}=\beta \mathbf{u}_{1} \wedge \cdots \wedge \mathbf{u}_{\sharp \mathfrak{u}}
$$

for some nonzero scalar $\beta$. If, utilizing the Clifford product,

$$
\mathfrak{u}=\alpha \mathbf{u}_{1} \cdots \mathbf{u}_{\sharp \mathfrak{u}}
$$

for some scalar $\alpha$, then the collection $\mathfrak{U}$ is said to constitute a Clifford factorization of $\mathfrak{u}$.

The term blade factorization refers to any process by which the constituent vectors of a $k$-blade are recovered. Such factorizations are obviously not unique.

The reversion on $\mathcal{C} \ell_{Q}(V)$ is defined on arbitrary blade $\mathfrak{u}=\mathbf{u}_{1} \cdots \mathbf{u}_{\sharp \mathfrak{u}}$ by

$$
\tilde{\mathfrak{u}}:=\mathbf{u}_{\sharp u} \cdots \mathbf{u}_{1}=(-1)^{\sharp \mathfrak{u}(\sharp \mathfrak{u}-1) / 2} \mathfrak{u},
$$

and is extended linearly to all of $\mathcal{C} \ell_{Q}(V)$. Similarly, the grade involution is defined by linear extension of $\hat{\mathfrak{u}}:=(-1)^{\sharp \mathfrak{u}} \mathfrak{u}$, and Clifford conjugation is defined as the composition of reversion and grade involution. Specifically, Clifford conjugation acts on an arbitrary blade $\mathfrak{u}$ according to $\overline{\mathfrak{u}}:=(-1)^{\sharp \mathfrak{u}(\sharp \mathfrak{u}+1) / 2} \mathfrak{u}$.

By utilizing reversion, the inner product $\langle\cdot, \cdot\rangle_{Q}$ is seen to extend to the full algebra $\mathcal{C} \ell_{Q}(V)$ by bilinear linear extension of

$$
\left\langle\mathfrak{b}_{1}, \mathfrak{b}_{2}\right\rangle_{Q}:=\left\langle\mathfrak{b}_{1} \widetilde{\mathfrak{b}_{2}}\right\rangle_{0}
$$

for arbitrary basis blades $\mathfrak{b}_{1}, \mathfrak{b}_{2}$.

Given the Clifford product, the left contraction operator is now conveniently defined for vector $\mathbf{x}$ and arbitrary multivector $v \in \mathcal{C} \ell_{Q}(V)$ by linear extension of

$$
\mathbf{x} v=\mathbf{x}\lrcorner v+\mathbf{x} \wedge v .
$$

A similar definition holds for the right contraction, i.e., $u \mathbf{x}:=u\llcorner\mathbf{x}+u \wedge \mathbf{x}$. The left and right contraction operators then extend associatively to blades 
and linearly to arbitrary elements $u, v \in \mathcal{C} \ell_{Q}(V)$. Moreover, left and right contractions are dual to the exterior product and satisfy the following:

$$
\begin{aligned}
\langle u\lrcorner v, w\rangle_{Q} & =\langle v, \tilde{u} \wedge w\rangle_{Q}, \\
\left\langle u\llcorner v, w\rangle_{Q}\right. & =\langle u, w \wedge \tilde{v}\rangle_{Q} .
\end{aligned}
$$

\subsection{Clifford operator calculus}

In the author's earlier joint work, the development of Clifford operator calculus (OC) was based on polynomial operator calculus [23, 24]. To begin, raising and lowering operators were defined naturally in terms of polynomial differentiation

and integration operators on Clifford multivectors regarded as polynomials in anticommuting variables. The Clifford differentiation operator $\partial / \partial \mathbf{x}$ is defined for anisotropic $\mathbf{x} \in V$ by linear extension of

$$
\left.\frac{\partial}{\partial \mathbf{x}} u=\mathbf{x}\right\lrcorner u .
$$

Combinatorial Clifford integrals are defined by

$$
\begin{gathered}
\int \mathrm{d} \mathbf{x}=\mathbf{x}, \\
\iint \mathrm{d} \mathbf{x} \mathrm{d} \mathbf{y}=\int \mathbf{x} \mathrm{d} \mathbf{y}=\mathbf{x} \wedge \mathbf{y} .
\end{gathered}
$$

The integrals here differ slightly from those defined in earlier works [22, 25]. In particular, the integrals here are suitable for defining right raising operators, rather than relying always on actions from the left. As should be the case in quantum probability, no bias is given to left over right.

These polynomial operators induce combinatorial raising and lowering operators by which Clifford monomials (blades) are "raised" from grade $k$ to grade $k+1$ or "lowered" from grade $k$ to grade $k-1$. These raising and lowering operators can also be regarded as fermion creation and annihilation operators in the sense of quantum mechanics.

For notational convenience, the product signature map $\vartheta: 2^{[n]} \times 2^{[n]} \rightarrow\{ \pm 1\}$ is defined naturally in terms of a counting measure on finite sets. Specifically, for fixed positive integer $j$, define the map $\mu_{j}: 2^{[n]} \rightarrow \mathbb{N}_{0}$ by

$$
\mu_{j}(I):=\sharp\{i \in I: i>j\} .
$$

Thus, the map $\mu_{j}(I)$ gives the counting measure of the set $\{i \in I: i>j\}$. Now, for multi-indices $I, J \in 2^{[n]}$, defining

$$
\vartheta(I, J)=(-1)^{\left(\sum_{j \in J} \mu_{j}(I)\right)} \prod_{\ell \in I \cap J}\left\langle\mathbf{x}_{\ell}, \mathbf{x}_{\ell}\right\rangle_{Q}
$$

gives $\mathbf{x}_{I} \mathbf{x}_{J}=\vartheta(I, J) \mathbf{x}_{I \triangle J}$, where $I \triangle J=(I \cup J) \backslash(I \cap J)$ denotes the setsymmetric difference of $I$ and $J$. 
Significantly, given a $Q$-orthogonal basis $\left\{\mathbf{x}_{j}: 1 \leq j \leq n\right\}$ of $V$, the exterior product and left contraction act as combinatorial raising and lowering operators on multi-indices of blades in $\mathcal{C} \ell_{Q}(V)$ :

$$
\mathbf{x}_{I} \wedge \mathbf{x}_{j}=\left\langle\mathbf{x}_{I} \mathbf{x}_{j}\right\rangle_{|I|+1}= \begin{cases}\vartheta(I,\{j\}) \mathbf{x}_{I \cup\{j\}} & \text { if } j \notin I, \\ 0 & \text { otherwise }\end{cases}
$$

and

$$
\left.\mathbf{x}_{j}\right\lrcorner \mathbf{x}_{I}=\left\langle\mathbf{x}_{j} \mathbf{x}_{I}\right\rangle_{|I|-1}= \begin{cases}\vartheta(\{j\}, I) \mathbf{x}_{I \backslash\{j\}} & \text { if } j \in I, \\ 0 & \text { otherwise. }\end{cases}
$$

For any anisotropic vector $\mathbf{x} \in \mathcal{C} \ell_{Q}(V)$, one can now define corresponding lowering and raising operators on $\mathcal{C} \ell_{Q}(V)$.

Definition 1.2. Let $\mathbf{x}$ be an anisotropic vector in $\mathcal{C} \ell_{Q}(V)$, and define the (left) lowering operator $\Lambda_{\mathbf{x}}$ on $\mathcal{C} \ell_{Q}(V)$ by

$$
\left.\Lambda_{\mathbf{x}} u=\frac{\partial}{\partial \mathbf{x}} u=\mathbf{x}\right\lrcorner u
$$

for any $u \in \mathcal{C} \ell_{Q}(V)$.

The lowering operator $\Lambda_{\mathbf{x}}$ is correctly regarded as an operator taking elements of grade $k$ to elements of grade $k-1$ for $k=1, \ldots, n$.

Definition 1.3. Let $\mathbf{x}$ be an anisotropic vector in $\mathcal{C} \ell_{Q}(V)$ and define the corresponding (right) raising operator $\Xi_{\mathbf{x}}$ on $\mathcal{C} \ell_{Q}(V)$ by

$$
\Xi_{\mathbf{x}} u=\int u d \mathbf{x}=u \wedge \mathbf{x}
$$

for any $u \in \mathcal{C} \ell_{Q}(V)$.

It will be convenient to denote by $\hat{\Xi}_{\mathbf{x}}$ and $\hat{\Lambda}_{\mathbf{x}}$ the left raising and right lowering operators, respectively. The role of raising and lowering operators in the Clifford product is made explicit by considering left and right multiplication by a vector, $\mathbf{x}$. Specifically, for $u \in \mathcal{C} \ell_{Q}(V)$, these are the operator sums

$$
\mathbf{x} u=\left(\hat{\Xi}_{\mathbf{x}} \oplus \Lambda_{\mathbf{x}}\right) u,
$$

and

$$
u \mathbf{x}=\left(\Xi_{\mathbf{x}} \oplus \hat{\Lambda}_{\mathbf{x}}\right) u .
$$

The relationship between the raising and lowering operators is made clear by the next lemma.

Lemma 1.4. For fixed anisotropic vector $\mathbf{x}$ in $\mathcal{C} \ell_{Q}(V)$, the operators $\Xi_{\mathbf{x}}, \Lambda_{\mathbf{x}}$, $\hat{\Xi}_{\mathbf{x}}$, and $\hat{\Lambda}_{\mathbf{x}}$ satisfy the following duality relations with respect to the inner product $\langle\cdot, \cdot\rangle_{Q}$ :

$$
\begin{aligned}
\left\langle\Lambda_{\mathbf{x}} u, w\right\rangle_{Q} & =\left\langle u, \hat{\Xi}_{\mathbf{x}} w\right\rangle_{Q}, \\
\left\langle\hat{\Lambda}_{\mathbf{x}} u, w\right\rangle_{Q} & =\left\langle u, \Xi_{\mathbf{x}} w\right\rangle_{Q},
\end{aligned}
$$

for all $u, w \in \mathcal{C} \ell_{Q}(V)$. 
Proof. The result follows immediately from (1.20) and the definitions of the lowering and raising operators.

Defining the composition of lowering (differential) operators as multivector left contraction operators also makes sense. Specifically, for fixed grade- $k$ basis blade $\mathbf{x}_{J}$ and arbitrary basis blade $\mathbf{x}_{I}$,

$$
\left.\left.\left.\left.\frac{\partial}{\partial \mathbf{x}_{j_{1}}} \cdots \frac{\partial}{\partial \mathbf{x}_{j_{k}}} \mathbf{x}_{I}=\mathbf{x}_{j_{1}}\right\lrcorner(\cdots\lrcorner\left(\mathbf{x}_{j_{k}}\right\lrcorner \mathbf{x}_{I}\right)\right):=\mathbf{x}_{J}\right\lrcorner \mathbf{x}_{I}
$$

As a consequence,

$$
\left.\mathbf{x}_{J}\right\lrcorner \mathbf{x}_{I}:= \begin{cases}\vartheta(J, I) \mathbf{x}_{I \backslash J} & \text { if } J \subseteq I, \\ 0 & \text { otherwise. }\end{cases}
$$

This multivector contraction operator extends linearly to all of $\mathcal{C} \ell_{Q}(V)$. This contraction operator is correctly regarded as a $k^{\text {th }}$ order lowering operator

$$
\Lambda_{\mathbf{x}_{J}} \mathbf{x}_{I}=\vartheta(J, I) \mathbf{x}_{I \backslash J} \in\left\langle\mathcal{C} \ell_{Q}(V)\right\rangle_{|I|-|J|} .
$$

One defines multivector right contraction operators in similar fashion.

Due to associativity, the exterior product already has a natural generalization; i.e.,

$$
\mathbf{x}_{I} \wedge \mathbf{x}_{J}=\mathbf{x}_{i_{1}} \wedge \cdots \wedge \mathbf{x}_{i_{|I|}} \wedge \mathbf{x}_{j_{1}} \wedge \cdots \wedge \mathbf{x}_{j_{|J|}}
$$

Consequently,

$$
\mathbf{x}_{I} \wedge \mathbf{x}_{J}= \begin{cases}\vartheta(I, J) \mathbf{x}_{I \cup J} & \text { if } I \cap J=\emptyset \\ 0 & \text { otherwise. }\end{cases}
$$

Note that the general blade product $\mathbf{x}_{I} \mathbf{x}_{J}$ has the operator calculus formulation

$$
\mathbf{x}_{I} \mathbf{x}_{J}=\left(\Lambda_{\mathbf{x}_{i_{1}}}+\Xi_{\mathbf{x}_{i_{1}}}\right) \circ \cdots \circ\left(\Lambda_{\mathbf{x}_{i_{|I|}}}+\Xi_{\mathbf{x}_{i_{|I|}}}\right) \mathbf{x}_{J}
$$

Lemma 1.5. For any anisotropic vector $\mathbf{x} \in \mathcal{C} \ell_{Q}(V)$, the corresponding lowering and raising operators are nilpotent of index 2. That is,

$$
\begin{aligned}
& \Lambda_{\mathbf{x}}^{2}:=\Lambda_{\mathbf{x}} \circ \Lambda_{\mathbf{x}}=0, \\
& \Xi_{\mathbf{x}}{ }^{2}:=\Xi_{\mathbf{x}} \circ \Xi_{\mathbf{x}}=0 .
\end{aligned}
$$

Further,

$$
\Lambda_{\mathbf{x}} \circ \hat{\Lambda}_{\mathbf{x}}=0=\Xi_{\mathbf{x}} \circ \hat{\Xi}_{\mathbf{x}} .
$$

Moreover, extending the operators associatively, via composition, to operators $\Lambda_{\mathfrak{x}}$ and $\Xi_{\mathfrak{x}}$ for an arbitrary blade $\mathfrak{x} \in \mathcal{C} \ell_{Q}(V)$,

$$
\Lambda_{\mathfrak{x}} \circ \hat{\Lambda}_{\mathfrak{x}-1}=0=\Xi_{\mathfrak{x}} \circ \hat{\Xi}_{\mathfrak{x}^{-1}}
$$

Proof. The result follows immediately from the properties of contractions and exterior products. 


\section{Endomorphisms of $V$ and $\mathcal{C} \ell_{Q}(V)$}

Beginning with a $Q$-orthogonal set of vectors $\left\{\mathbf{u}_{1}, \ldots, \mathbf{u}_{\ell}\right\} \subset V$, their product is an $\ell$-blade, $\mathfrak{u} \in \mathcal{C} \ell_{Q}(V)$. Define the mapping $\varphi_{\mathfrak{u}}: \mathcal{C} \ell_{Q}(V) \rightarrow \mathcal{C} \ell_{Q}(V)$ by

$$
\varphi_{\mathfrak{u}}(x):=\mathfrak{u} x \frac{\tilde{\mathfrak{u}}}{\mathfrak{u} \tilde{\mathfrak{u}}}=\mathfrak{u} x \mathfrak{u}^{-1} .
$$

For a fixed blade $\mathfrak{u}$, the linear map $x \mapsto \mathfrak{u} x \mathfrak{u}^{-1}$ is an endomorphism on $\mathcal{C} \ell_{Q}(V)$ referred to as the conjugation of $x$ by the blade $\mathfrak{u}$, or simply as a blade conjugation.

Lemma 2.1. Given a blade $\mathfrak{u} \in \mathcal{C} \ell_{Q}(V)$, the operator $\varphi_{\mathfrak{u}} \in \mathcal{L}\left(\mathcal{C} \ell_{Q}(V)\right)$ defined by $x \mapsto \mathfrak{u} x \mathfrak{u}^{-1}$ has the operator calculus representation

$$
\varphi_{\mathfrak{u}} \simeq \Lambda_{\mathfrak{u}} \Xi_{\mathfrak{u}^{-1}}+\hat{\Xi}_{\mathfrak{u}} \hat{\Lambda}_{\mathfrak{u}^{-1}} .
$$

Proof. In light of (1.31), (1.32), and Lemma 1.5,

$$
\begin{aligned}
\varphi_{\mathfrak{u}}=\left(\Lambda_{\mathfrak{u}} \oplus \hat{\Xi}_{\mathfrak{u}}\right)\left(\hat{\Lambda}_{\mathfrak{u}-1} \oplus \Xi_{\mathfrak{u}^{-1}}\right) & \\
=\Lambda_{\mathfrak{u}} \hat{\Lambda}_{\mathfrak{u}^{-1}}+\hat{\Xi}_{\mathfrak{u}} \Xi_{\mathfrak{u}^{-1}}+\Lambda_{\mathfrak{u}} \Xi_{\mathfrak{u}^{-1}} & +\hat{\Xi}_{\mathfrak{u}} \hat{\Lambda}_{\mathfrak{u}^{-1}} \\
& =\Lambda_{\mathfrak{u}} \Xi_{\mathfrak{u}^{-1}}+\hat{\Xi}_{\mathfrak{u}} \hat{\Lambda}_{\mathfrak{u}^{-1}}
\end{aligned}
$$

A thorough study of the operator $\varphi_{\mathfrak{u}}$ begins with the restriction $\Phi_{\mathfrak{u}}=\left.\varphi_{\mathfrak{u}}\right|_{V}$.

When $\Phi_{\mathfrak{u}}$ is an endomorphism on $V$ having eigenvalue $\lambda$, let $\mathcal{E}_{\lambda}$ denote the corresponding eigenspace. A blade test is now given by the following theorem.

Theorem 2.2. A homogeneous, grade- $k$ multivector $\mathfrak{u} \in \mathcal{C} \ell_{Q}(V)$ is a blade if and only if $\Phi_{\mathfrak{u}}$ is an endomorphism on $V$ with eigenvalues $\lambda_{1}=(-1)^{k-1}$ and $\lambda_{2}=-\lambda_{1}=(-1)^{k}$ such that

$$
\begin{gathered}
\operatorname{dim}\left(\mathcal{E}_{\lambda_{1}}\right)=k, \\
\operatorname{dim}\left(\mathcal{E}_{\lambda_{2}}\right)=n-k .
\end{gathered}
$$

Proof. First, if $\mathfrak{u}=\mathbf{u}_{1} \cdots \mathbf{u}_{k}$ is a blade, invertibility of $\mathfrak{u}$ follows from

$$
\mathfrak{u} \tilde{\mathfrak{u}}=\mathbf{u}_{1} \cdots \mathbf{u}_{k} \mathbf{u}_{k} \cdots \mathbf{u}_{1}=\prod_{i=1}^{k}\left\langle\mathbf{u}_{i}, \mathbf{u}_{i}\right\rangle_{Q} \in \mathbb{R} \neq 0 .
$$

Whence, $\mathfrak{u}^{-1}=\frac{\tilde{\mathfrak{u}}}{\mathfrak{u} \tilde{\mathfrak{u}}}$. Decomposing an arbitrary vector $\mathrm{x} \in V$ into components parallel and orthogonal to $\mathbf{u}_{k}$,

$$
\begin{aligned}
\mathbf{u}_{k} \mathbf{x} \mathbf{u}_{k}=\mathbf{u}_{k}\left(\mathbf{x}_{\|}+\mathbf{x}_{\perp}\right) \mathbf{u}_{k}=\left\langle\mathbf{x}_{\|}, \mathbf{u}_{k}\right\rangle_{Q} \mathbf{u}_{k}-\left\langle\mathbf{u}_{k}, \mathbf{u}_{k}\right\rangle_{Q} \mathbf{x}_{\perp} & \\
& =\alpha \mathbf{x}_{\|}+\beta \mathbf{x}_{\perp} \in V,
\end{aligned}
$$


for scalars $\alpha$ and $\beta$. Associative extension gives $\mathfrak{u x} \tilde{\mathfrak{u}} \in V$, so that $\Phi_{\mathfrak{u}}: V \rightarrow V$ is a well-defined linear operator. Invertibility of $\Phi_{\mathfrak{u}}$ follows from invertibility of u. Hence, $\Phi_{\mathfrak{u}}^{-1}=\Phi_{\mathfrak{u}^{-1}}$.

Keep in mind that the factors of $\mathfrak{u}$ are unique only up to isomorphism, i.e., change of basis. Writing $u=\mathbf{x}_{1} \cdots \mathbf{x}_{k}$ for $Q$-orthogonal anisotropic vectors $\left\{\mathbf{x}_{i}: 1 \leq i \leq n\right\}$ and choosing any factor $\mathbf{x}_{i}$, it follows immediately that

$$
\begin{aligned}
\Phi_{\mathfrak{u}}\left(\mathbf{x}_{i}\right)=\frac{1}{\mathfrak{u} \tilde{\mathfrak{u}}} \mathbf{x}_{1} \cdots \mathbf{x}_{k} \mathbf{x}_{i} \mathbf{x}_{k} \cdots \mathbf{x}_{1} \\
=\frac{(-1)^{k-i} Q\left(\mathbf{x}_{i}\right)(-1)^{i-1}}{\mathfrak{u} \tilde{\mathfrak{u}}} \mathbf{x}_{i} \prod_{j \neq i} Q\left(\mathbf{x}_{k}\right) \\
=\frac{(-1)^{k-1}}{\mathfrak{u} \tilde{\mathfrak{u}}} \mathbf{x}_{i} \prod_{1 \leq j \leq k} Q\left(\mathbf{x}_{j}\right) \\
\quad=\frac{(-1)^{k-1}}{\mathfrak{u} \tilde{\mathfrak{u}}} \mathbf{x}_{i} \mathfrak{u} \tilde{\mathfrak{u}}=(-1)^{k-1} \mathbf{x}_{i}
\end{aligned}
$$

Hence, $\left\{\mathbf{x}_{i}: 1 \leq i \leq k\right\}$ is a basis for the eigenspace $\mathcal{E}_{\lambda_{1}}$.

Further, if $\mathbf{v}$ is $Q$-orthogonal to $\mathcal{E}_{\lambda_{1}}$, then

$$
\begin{aligned}
& \Phi_{\mathfrak{u}}(\mathbf{v})=\frac{1}{\mathfrak{u} \tilde{\mathfrak{u}}} \mathbf{x}_{1} \cdots \mathbf{x}_{k} \mathbf{v} \mathbf{x}_{k} \cdots \mathbf{x}_{1} \\
&=\frac{(-1)^{k}}{\mathfrak{u} \tilde{\mathfrak{u}}} \mathbf{v} \prod_{1 \leq j \leq k} Q\left(\mathbf{x}_{j}\right) \\
& \quad=\frac{(-1)^{k} \mathfrak{u} \tilde{\mathfrak{u}}}{\mathfrak{u} \tilde{\mathfrak{u}}} \mathbf{v}=(-1)^{k} \mathbf{v}
\end{aligned}
$$

Hence, $\mathbf{v}$ is an eigenvector of $\varphi_{\mathfrak{u}}$ associated with eigenvalue $\lambda_{2}=(-1)^{k}$. Since $\mathbf{v}$ was arbitrarily chosen from the orthogonal complement of $\mathcal{E}_{\lambda_{1}}$, it follows that $\operatorname{dim}\left(\mathcal{E}_{\lambda_{2}}\right)=n-k$.

Conversely, suppose $\Phi$ is a linear operator on $V$ with the prescribed eigenvalues and eigenspaces, and let $\left\{\mathbf{u}_{j}: 1 \leq j \leq k\right\}$ be an arbitrary $Q$-orthogonal basis of $\mathcal{E}_{\lambda_{1}}$. Setting $\mathfrak{u}=\prod_{1 \leq j \leq k} \mathbf{u}_{j}$, it is clear that $\mathfrak{u}$ is an invertible product of $Q$-orthogonal vectors. Hence, $\mathfrak{u}$ is a $k$-blade. By the previous arguments, the action on $V$ of conjugation by $\mathfrak{u}$, i.e., $\mathbf{x} \mapsto \mathfrak{u x} \mathfrak{u}^{-1}$, is exactly the action of $\Phi$ on $V$. Therefore, $\Phi=\Phi_{\mathfrak{u}}$.

Considering conjugation by a blade $\mathfrak{u}$, we adopt the notational convention

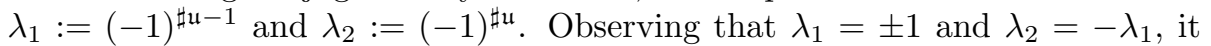
is clear that $\Phi_{\mathfrak{u}}$ acts as the identity on $\mathcal{E}_{+1}$ and as a reflection (or reversal) on $\mathcal{E}_{-1}$. It follows immediately that $\Phi_{\mathfrak{u}}$ is an involution; i.e., $\Phi_{\mathfrak{u}}{ }^{2}=\mathcal{I}$.

The collection of all $Q$-orthogonal transformations on $V$ forms a group called the orthogonal group of $Q$, denoted $\mathrm{O}_{Q}(V)$. Specifically, $T \in \mathrm{O}_{Q}(V)$ if and only if for every $\mathbf{x} \in V, Q(T(\mathbf{x}))=Q(\mathbf{x})$. The conformal orthogonal group, 
denoted $\mathrm{CO}_{Q}(V)$, is the direct product of the orthogonal group with the group of dilations. More specifically, $\tau \in \mathrm{CO}_{Q}(V)$ if and only if for every $\mathbf{x} \in V$, there exists a scalar $\lambda$ such that $Q(\tau(\mathbf{x}))=\lambda^{2} Q(\mathbf{x})$.

It is not difficult to see that blade conjugation is an orthogonal transformation on $V$. The next lemma makes this formal.

Lemma 2.3. Let $\mathfrak{u} \in \mathcal{C} \ell_{Q}(V)$ be a blade. Then, $\Phi_{\mathfrak{u}} \in \mathrm{O}_{Q}(V)$.

Proof. The result is established by showing that the eigenspaces $\mathcal{E}_{\lambda_{1}}$ and $\mathcal{E}_{\lambda_{2}}$ are orthogonal with respect to the quadratic form $Q$. Begin by letting $\mathbf{v} \in \mathcal{E}_{\lambda_{1}}$ and $\mathbf{w} \in \mathcal{E}_{\lambda_{2}}$. Then,

$$
\mathfrak{u} \mathbf{v} u^{-1} \mathfrak{u} \mathbf{w} u^{-1}=\lambda_{1} \lambda_{2} \mathbf{v w}=\lambda_{1} \lambda_{2}\left(\langle\mathbf{v}, \mathbf{w}\rangle_{Q}+\mathbf{v} \wedge \mathbf{w}\right) .
$$

Letting $\alpha=\langle\mathbf{v}, \mathbf{w}\rangle_{Q}$ and noting that $\lambda_{1} \lambda_{2}=-1$, this implies

$$
\mathfrak{u}(\mathbf{v w}) \mathfrak{u}^{-1}=-\alpha-\mathbf{v} \wedge \mathbf{w} .
$$

On the other hand, noting that $\mathbf{v w}=\alpha+\mathbf{v} \wedge \mathbf{w}$, one also finds

$$
\mathfrak{u}(\mathbf{v w}) \mathfrak{u}^{-1}=\mathfrak{u}(\alpha+\mathbf{v} \wedge \mathbf{w}) \mathfrak{u}^{-1}=\alpha-\mathbf{v} \wedge \mathbf{w} .
$$

Equality of (2.9) and (2.10) then implies $\alpha=0$.

Given an arbitrary $k$-blade $\mathfrak{u}$, one now readily defines the level- $\ell$ induced $\operatorname{map} \varphi_{\mathfrak{u}}{ }^{(\ell)}$ on blades of grade $\ell>1$ by

$$
\varphi_{\mathfrak{u}}^{(\ell)}\left(\mathbf{w}_{1} \ldots \mathbf{w}_{\ell}\right):=\mathfrak{u} \prod_{j=1}^{\ell} \mathbf{w}_{j} \mathfrak{u}^{-1}=\prod_{j=1}^{\ell} \mathfrak{u} \mathbf{w}_{j} \mathfrak{u}^{-1}=\prod_{j=1}^{\ell} \Phi_{\mathfrak{u}}\left(\mathbf{w}_{j}\right) .
$$

As an immediate consequence of Lemma 2.3, the induced map is a well-defined invertible linear transformation on the grade- $\ell$ subspace $\bigwedge^{\ell} V$ of $\mathcal{C} \ell_{Q}(V)$; that is, the image of an $\ell$-blade is also an $\ell$-blade. In fact, the induced map is the restriction of $\varphi_{\mathfrak{u}}$ to the grade- $\ell$ subspace of $\mathcal{C} \ell_{Q}(V)$ :

$$
\varphi_{\mathfrak{u}}^{(\ell)}=\left.\varphi_{\mathfrak{u}}\right|_{\Lambda^{\ell} V} .
$$

Given a blade $\mathfrak{u}$, a useful generalization of the conjugation operator is defined by setting

$$
\psi_{\mathfrak{u}}(x):=\mathfrak{u} x \tilde{\mathfrak{u}}
$$

for $x \in \mathcal{C} \ell_{Q}(V)$. This linear map $x \mapsto \mathfrak{u} x \tilde{u}$ is an endomorphism on $\mathcal{C} \ell_{Q}(V)$ referred to as generalized conjugation of $x$ by the blade $\mathfrak{u}$, or simply as a generalized blade conjugation. When, $\mathfrak{u} \tilde{\mathfrak{u}}=1$, one sees that $\varphi_{\mathfrak{u}}=\psi_{\mathfrak{u}}$.

For convenience, the restriction of $\psi_{\mathfrak{u}}$ to $V$ is denoted by $\Psi_{\mathfrak{u}}$. Properties of $\Psi_{\mathfrak{u}}$ differ from those of $\Phi_{\mathfrak{u}}$ in scaling. In particular, $\Psi_{\mathfrak{u}}$ is an element of the 
conformal orthogonal group, $\mathrm{CO}_{Q}(V)$. Applying the reasoning used in the proof of Theorem 2.2 reveals that

$$
\Psi_{\mathfrak{u}}(\mathbf{v})= \begin{cases}(-1)^{\sharp \mathfrak{u}-1} \mathfrak{u} \tilde{u} \mathbf{v} & \text { if } \mathbf{v} \mid \mathfrak{u}, \\ (-1)^{\sharp \mathfrak{u}} \mathfrak{u} \tilde{u} \mathbf{v} & \text { if } \mathbf{v} \mid \mathfrak{u}^{\star} .\end{cases}
$$

Consequently,

$$
\Psi_{\mathfrak{u}}=(\mathfrak{u} \tilde{\mathfrak{u}}) \Phi_{\mathfrak{u}} .
$$

Remark 2.4. The geometric significance of the conjugation $\Phi_{\mathfrak{u}}(x)=\mathfrak{u x u}^{-1}$ is well explained in the paper by Lounesto and Latvamaa [18]. For example, when $Q$ is the quadratic form of signature $(p, q)$ corresponding to the Clifford algebra $\mathcal{C} \ell_{p, q}$ and $\mathfrak{u} \tilde{\mathfrak{u}}=1$, the following exact sequences exist:

$$
\begin{aligned}
1 & \rightarrow \mathbb{Z}_{2} \rightarrow \operatorname{Pin}(p, q) \stackrel{\Phi_{u}}{\rightarrow} \mathrm{O}(p, q) \rightarrow 1, \\
1 & \rightarrow \mathbb{Z}_{2} \rightarrow \operatorname{Spin}(p, q) \stackrel{\Phi_{u}}{\rightarrow} \mathrm{SO}(p, q) \rightarrow 1 .
\end{aligned}
$$

Here, $\operatorname{Pin}(p, q)$ and $\operatorname{Spin}(p, q)$ are the Pin and Spin groups, which constitute double coverings of the corresponding orthogonal groups. The irreducible representations of these groups are pinors and spinors.

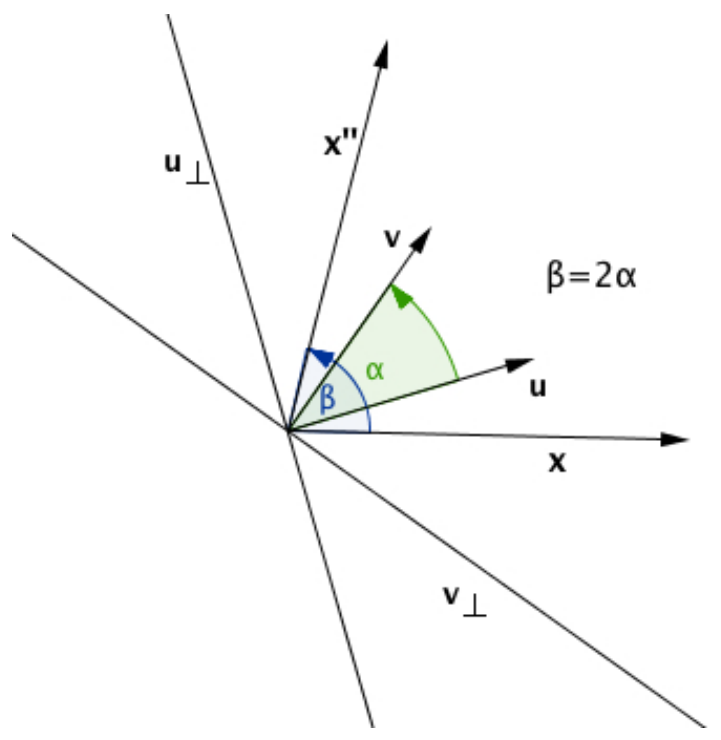

Figure 2.1: Rotation from two hyperplane reflections.

Corollary 2.5. Given a unit blade $\mathfrak{u} \in \mathcal{C} \ell_{Q}(V)$, where $Q$ is positive definite, the map $\mathbf{x} \mapsto \mathfrak{u x u}^{-1}$ represents a composition of hyperplane reflections across pairwise-orthogonal hyperplanes. In particular, when $\sharp \mathfrak{u}$ is even, the mapping 
is a composition of rotations by $\pi$ radians in each of $\sharp \mathfrak{u} / 2$ orthogonal planes. When $n \equiv \sharp \mathfrak{u} \equiv 1(\bmod 2)$, the mapping is a composition of $\pi$ radian rotations in $(n-\sharp \mathfrak{u}) / 2$ orthogonal planes. When $n \equiv 0(\bmod 2)$ and $\sharp \mathfrak{u} \equiv 1(\bmod 2)$, the mapping is a composition of $\pi$ radian rotations in $(n-\sharp \mathfrak{u}-1) / 2$ orthogonal planes along with one reflection through an additional orthogonal hyperplane.

Proof. To begin, a well known geometric result is recalled. Let $\mathbf{u}, \mathbf{v}$ be unit vectors separated by angle $\alpha$ (measured from $\mathbf{u}$ to $\mathbf{v}$ ), and let $\mathbf{x} \in V$ be arbitrary. The mapping $\mathbf{x} \mapsto \mathbf{x}^{\prime \prime}$ defined by the composition of reflections across hyperplanes orthogonal to $\mathbf{u}$ and $\mathbf{v}$, respectively, corresponds to rotation by angle $2 \alpha$ in the uv-plane, as pictured in Figure 2.1.

Letting $\mathfrak{u}$ be a blade of grade $k$, the proof of Theorem 2.2 shows that for any unit vector $\mathbf{v} \in \mathcal{E}_{\left.(-1)^{1+(\sharp u}(\bmod 2)\right)}$, the product $-\mathbf{v x v}$ is the reflection of $\mathbf{x}$ across the hyperplane orthogonal to $\mathbf{v}$. Orthogonality of the constituent vectors of $\mathfrak{u}$ then guarantees that the map $\mathbf{x} \mapsto \mathfrak{u x u ^ { - 1 }}$ represents a composition of reflections across pairwise orthogonal hyperplanes. The composition of any two such reflections thereby results in rotation by $\pi$ radians.

The next definition addresses how a vector can be said to "divide" a blade.

Definition 2.6. Let $\mathfrak{u}$ be a blade in $\mathcal{C} \ell_{Q}(V)$. A vector $\mathbf{w} \in V$ is said to divide $\mathfrak{u}$ if and only if there exists a blade $\mathfrak{u}^{\prime} \in \mathcal{C} \ell_{Q}(V)$ of grade $\sharp \mathfrak{u}-1$ such that $\mathfrak{u}= \pm \mathbf{w} \mathfrak{u}^{\prime}$. In this case, one writes $\mathbf{w} \mid \mathfrak{u}$.

Remark 2.7. An important interpretation of Theorem 2.2 is that any $Q$-orthogonal basis of the eigenspace $\mathcal{E}_{\lambda_{1}}$ of $\Phi_{\mathfrak{u}}$ determines a Clifford factorization of $\mathfrak{u}$. Moreover, any $Q$-orthogonal basis of $\mathcal{E}_{\lambda_{2}}$ determines a Clifford factorization of the dual blade $\mathfrak{u}^{\star}[22]$.

\subsection{Quantum Random Variables and States of the Clifford Algebra}

In quantum probability, self-adjoint operators are analogous to random variables, with the expectation being given by the operator's normalized trace. Good references for the underlying theory include books by Gudder [15], Meyer [20], and Parthasarathy [21].

The role played by Kravchuk polynomials and Kravchuk matrices in quantum probability has been studied in a number of papers by Feinsilver et al. [12, 10]. Spectral properties of symmetric Kravchuk matrices, which are clearly self-adjoint, have also been considered in work by Feinsilver and Fitzgerald [9].

When $\mathcal{A}$ is a complex algebra with involution $*$ and unit 1 , a positive linear *-functional on $\mathcal{A}$ satisfying $\varphi(1)=1$ is called a state on $\mathcal{A}$. The pair $(\mathcal{A}, \varphi)$ is called an algebraic probability space.

As seen in Lemma 2.3, $\Phi_{\mathfrak{u}}$ is a $Q$-orthogonal transformation on $V$. An immediate consequence is that $\Phi_{\mathfrak{u}}$ is self-adjoint with respect to the $Q$-inner product, as made formal in the following lemma. 
Lemma 2.8. The blade conjugation operator $\Phi_{\mathfrak{u}}$ is self-adjoint with respect to $\langle\cdot, \cdot\rangle_{Q} \cdot$

Proof. Let $\mathbf{x}, \mathbf{y} \in V$ be arbitrary, and decompose $\mathbf{x}, \mathbf{y}$ into components lying in $\mathcal{E}_{\lambda_{1}}$ and $\mathcal{E}_{\lambda_{2}}$. Then

$$
\begin{aligned}
\left\langle\mathbf{x}, \Phi_{\mathfrak{u}}(\mathbf{y})\right\rangle_{Q} & =\left\langle\left(\mathbf{x}_{1}+\mathbf{x}_{2}\right) \widetilde{\Phi_{\mathfrak{u}}(\mathbf{y})}\right\rangle_{0} \\
& =\left\langle\left(\mathbf{x}_{1}+\mathbf{x}_{2}\right)\left((-1)^{\sharp \mathfrak{u}-1} \mathbf{y}_{1}+(-1)^{\sharp \mathfrak{u}} \mathbf{y}_{2}\right)\right\rangle_{0} \\
& =\left\langle\mathbf{x}_{1}\left((-1)^{\sharp \mathfrak{u}-1} \mathbf{y}_{1}+(-1)^{\sharp \mathfrak{u}} \mathbf{y}_{2}\right)+\mathbf{x}_{2}\left((-1)^{\sharp \mathfrak{u}-1} \mathbf{y}_{1}+(-1)^{\sharp \mathfrak{u}} \mathbf{y}_{2}\right)\right\rangle_{0} \\
& =\left\langle\left((-1)^{\sharp \mathfrak{u}-1} \mathbf{x}_{1}+(-1)^{\sharp \mathfrak{u}} \mathbf{x}_{2}\right)\left(\mathbf{y}_{1}+\mathbf{y}_{2}\right)\right\rangle_{0} \\
& =\left\langle\mathfrak{u}\left(\mathbf{x}_{1}+\mathbf{x}_{2}\right) \mathfrak{u}^{-1}\left(\mathbf{y}_{1}+\mathbf{y}_{2}\right)\right\rangle_{0} \\
& =\left\langle\Phi_{\mathfrak{u}}(\mathbf{x}), \mathbf{y}\right\rangle_{Q} .
\end{aligned}
$$

Hence, $\Phi_{\mathfrak{u}}$ is correctly regarded as a quantum random variable. From the spectral properties uncovered in Theorem 2.2, one now interprets $\Phi_{\mathfrak{u}}$ as a quantum random variable $\xi$ taking values \pm 1 and satisfying

$$
\begin{gathered}
\mathbb{P}\left(\xi=\lambda_{1}\right)=\frac{\sharp \mathfrak{u}}{n}, \\
\mathbb{P}\left(\xi=\lambda_{2}\right)=\frac{n-\sharp \mathfrak{u}}{n} .
\end{gathered}
$$

The expectation, $\langle\xi\rangle=\operatorname{Tr}\left(\Phi_{\mathfrak{u}}\right)$, is thus seen to be

$$
\langle\xi\rangle=\frac{1}{n}\left((-1)^{\sharp \mathfrak{u}-1} \sharp \mathfrak{u}+(-1)^{\sharp \mathfrak{u}}(n-\sharp \mathfrak{u})\right)=\left\{\begin{array}{lll}
1-\frac{2 \sharp \mathfrak{u}}{n} & \sharp \mathfrak{u} \equiv 0 & (\bmod 2), \\
\frac{2 \sharp \mathfrak{u}}{n}-1 & \sharp \mathfrak{u} \equiv 1 & (\bmod 2) .
\end{array}\right.
$$

As Lemma 2.10 will show, the scalar projection $\langle\cdot\rangle_{0}: \mathcal{C} \ell_{Q}(V) \rightarrow \mathbb{R}$ defines a state on the Clifford algebra. For convenience, define the notation $\pi_{0}(u):=\langle u\rangle_{0}$. Further, when $Q$ is a definite quadratic form on $V$, one can ensure $u^{*} u \geq 0$ for arbitrary $u \in \mathcal{C} \ell_{Q}(V)$ and appropriate choice of involution. In this way one can formulate the additional requirement $\rho\left(u^{*} u\right) \geq 0$ for states of the probability space.

On the other hand, when $Q$ is indefinite, $\pi_{0}$ is still a state on $\mathcal{C} \ell_{Q}(V)$, but one cannot guarantee $u^{*} u \geq 0$ for all $u \in \mathcal{C} \ell_{Q}(V)$ using any of the canonical Clifford involutions. To address this potential shortcoming, the blade adjoint involution is defined.

Definition 2.9. Let $\mathfrak{u} \in \mathcal{C} \ell_{Q}(V)$ be a blade, and write $\mathfrak{u}=\prod_{i=1}^{\sharp \mathfrak{u}} \mathbf{u}_{i}$ for appropriately ordered vectors of $V$. Define the adjoint $\mathfrak{u}^{\dagger} \in \mathcal{C} \ell_{Q}(V)$ by

$$
\mathfrak{u}^{\dagger}:=\frac{\mathfrak{u} \tilde{\mathfrak{u}}}{|\mathfrak{u} \tilde{\mathfrak{u}}|} \tilde{\mathfrak{u}} .
$$


Clearly, $\left(\mathfrak{u}^{\dagger}\right)^{\dagger}=\mathfrak{u}$; i.e., $\dagger$ is an involution on blades of $\mathcal{C} \ell_{Q}(V)$ and extends linearly to the entire algebra.

Lemma 2.10. A state of the Clifford algebra $\mathcal{C} \ell_{Q}(V)$ is defined by the functional $\pi_{0}(u):=\langle u\rangle_{0}, u \in \mathcal{C} \ell_{Q}(V)$. For any nondegenerate quadratic form $Q$, the functional satisfies $\pi_{0}\left(u^{\dagger} u\right) \geq 0$ for all $u \in \mathcal{C} \ell_{Q}(V)$, where $\dagger$ denotes the blade adjoint defined by (2.19).

Proof. Direct computation shows that $\pi_{0}$ is a linear functional satisfying the required conditions.

Remark 2.11. In fact, when $Q$ is a definite quadratic form on $V$,

$$
u^{\dagger}= \begin{cases}\tilde{u} & \text { when } Q \text { is positive definite } \\ \bar{u} & \text { when } Q \text { is negative definite }\end{cases}
$$

Another formulation of combinatorial integrals, attributed to Berezin [3], proves useful. For purposes of the current paper, the following definition is established.

Definition 2.12. The Clifford Berezin integral of arbitrary $u \in \mathcal{C} \ell_{Q}(V)$ is defined for anisotropic $\mathrm{x} \in V$ by

$$
\int_{\mathfrak{B}} u d \mathbf{x}:=u\llcorner\mathbf{x} .
$$

Iterated Berezin integrals are defined by associative extension; i.e., for blade $\mathfrak{x}=\mathbf{x}_{1} \cdots \mathbf{x}_{\sharp \mathfrak{x}}$,

$$
\int_{\mathfrak{B}} u d \mathfrak{x}:=\left(\left(u \llcorner \mathbf { x } _ { 1 } ) \llcorner \cdots ) \left\llcorner\mathbf{x}_{\sharp \mathfrak{x}} .\right.\right.\right.
$$

Considering the iterated Clifford Berezin integral associated with the pseudo scalar, one obtains the following simplification:

$$
\int_{\mathfrak{B}} u d \omega:=\langle u \omega\rangle_{0} .
$$

One can now define a pseudo-state by making use of the pseudo scalar, $\omega$. The following lemma is obvious.

Lemma 2.13. The mapping $\rho_{\mathfrak{B}}(u):=\int_{\mathcal{B}} u d \omega: \mathcal{C} \ell_{Q}(V) \rightarrow \mathbb{R}$ is a positive linear functional on $\mathcal{C} \ell_{Q}(V)$ satisfying $\rho_{\mathfrak{B}}(\omega)=1$.

In quantum probability terms, blades represent pure states in $\mathcal{C} \ell_{Q}(V)$. Following the notation of Shale and Stinespring in their work on states of the Clifford algebra, one associates left and right operators with blades of the Clifford algebra [26] .

Given a blade $\mathfrak{u} \in \mathcal{C} \ell_{Q}(V)$, the operators $\mathfrak{L}_{\mathfrak{u}}$ and $\mathfrak{R}_{\mathfrak{u}}$ are defined on $\mathcal{C} \ell_{Q}(V)$ by $\mathfrak{L}_{\mathfrak{u}} v=\mathfrak{u} v$ and $\mathfrak{R}_{\mathfrak{u}} v=v \mathfrak{u}$, respectively. The map $\varphi_{\mathfrak{u}}$ thus satisfies

$$
\varphi_{\mathfrak{u}}=\mathfrak{L}_{\mathfrak{u}} \mathfrak{R}_{\mathfrak{u}^{-1}}=\mathfrak{R}_{\mathfrak{u}^{-1}} \mathfrak{L}_{\mathfrak{u}}
$$


The left-lowering operator $\Lambda_{\mathbf{x}}$ defined by $(1.29)$ on $\mathcal{C} \ell_{Q}(V)$ can now be expressed by

$$
\left.\Lambda_{\mathbf{x}} \mathfrak{u}:=\langle\mathbf{x} \mathfrak{u}\rangle_{\sharp \mathfrak{u}-1}=\left\langle\mathfrak{L}_{\mathbf{x}} \mathfrak{u}\right\rangle_{\sharp \mathfrak{u}-1}=\left(\pi_{\sharp \mathfrak{u}-1} \circ \mathfrak{L}_{\mathbf{x}}\right)(\mathfrak{u})=\mathbf{x}\right\lrcorner \mathfrak{u} .
$$

Similarly, the right-raising operator $\Xi_{\mathbf{x}}$ defined by $(1.30)$ on $\mathcal{C} \ell_{Q}(V)$ can be expressed by

$$
\Xi_{\mathbf{x}} \mathfrak{u}:=\langle\mathfrak{u x}\rangle_{\sharp \mathfrak{u}+1}=\left\langle\mathfrak{R}_{\mathbf{x}} \mathfrak{u}\right\rangle_{\sharp \mathfrak{u}+1}=\left(\pi_{\sharp \mathfrak{u}+1} \circ \mathfrak{R}_{\mathbf{x}}\right)(\mathfrak{u})=\mathfrak{u} \wedge \mathbf{x} .
$$

These extend associatively, via composition, to operators $\Lambda_{\mathfrak{x}}$ and $\Xi_{\mathfrak{x}}$ for arbitrary blade $\mathfrak{x} \in \mathcal{C} \ell_{Q}(V)$. It is easily verified that

$$
\varphi_{\mathfrak{u}}=\left[\frac{1}{2}\left(\mathfrak{L}_{\mathfrak{u}}+\mathfrak{R}_{\mathfrak{u}^{-1}}\right)\right]^{2}
$$

so that $\frac{1}{2}\left(\mathfrak{L}_{\mathfrak{u}}+\mathfrak{R}_{\mathfrak{u}^{-1}}\right)$ represents a "square root" of the conjugation operator $\varphi_{\mathfrak{u}}$, and hence a "fourth root of unity".

\subsection{Kravchuk polynomials from blade conjugation}

Discussion of the level-1 operator $\Phi_{\mathfrak{u}}$ now concludes with the recovery of Kravchuk polynomials from the operator's characteristic polynomial. Moreover, this characteristic polynomial is recovered from an iterated Berezin integral.

Theorem 2.14. Let $\mathfrak{u} \in \mathcal{C} \ell_{Q}(V)$ be a blade, let $\left\{\mathbf{e}_{\ell}: 1 \leq \ell \leq n\right\}$ be a fixed $Q$-orthonormal basis for $V$, and for each $\ell=1, \ldots, n$, define the Clifford polynomial $\xi_{\ell}(t)$ by

$$
\xi_{\ell}(t):=\Phi_{\mathfrak{u}}\left(\mathbf{e}_{\ell}\right)-t \mathbf{e}_{\ell} .
$$

If the collection $\left\{\xi_{\ell}(t): 1 \leq \ell \leq n\right\}$ is orthogonal, then the corresponding iterated Berezin integral is

$$
\int_{\mathfrak{B}} \omega \mathrm{d} \xi_{n}(t) \cdots \mathrm{d} \xi_{1}(t)= \begin{cases} \pm \sum_{\ell=0}^{n} K_{\ell}(n-\sharp \mathfrak{u}, n) t^{\ell} & \text { if } \sharp \mathfrak{u} \text { even, } \\ \pm \sum_{\ell=0}^{n} K_{\ell}(\sharp \mathfrak{u}, n) t^{\ell} & \text { if } \sharp \mathfrak{u} \text { odd. }\end{cases}
$$

Proof. With due consideration to (1.7), the exterior product satisfies

$$
\bigwedge_{\ell=1}^{n} \xi_{\ell}(t)=\bigwedge_{\ell=1}^{n}\left(\Phi_{\mathfrak{u}}\left(\mathbf{e}_{\ell}\right)-t \mathbf{e}_{\ell}\right)=\operatorname{det}\left(\Phi_{\mathfrak{u}}-t \mathcal{I}\right) \omega,
$$

where $\mathcal{I}$ is the identity on $V$. Reversing the order of the differentials reverses both the pseudoscalar and the computation of the determinant, resulting in an 
identity. Moreover, from the definition of the Berezin integral and the fact that $\omega\llcorner\mathfrak{z}=\omega \mathfrak{z}$ for any blade, $\mathfrak{z}$, one can rewrite the integral in the form

$$
\begin{aligned}
\int_{\mathfrak{B}} \omega \mathrm{d} \xi_{n}(t) \cdots \mathrm{d} \xi_{1}(t)=\left\langle\omega \left(\xi_{n}(t)\right.\right. & \left.\left.\wedge \cdots \wedge \xi_{1}(t)\right)\right\rangle_{0} \\
& =\left\langle\omega \operatorname{det}\left(\Phi_{\mathfrak{u}}-t \mathcal{I}\right) \omega\right\rangle_{0}=\omega^{2} \operatorname{det}\left(\Phi_{\mathfrak{u}}-t \mathcal{I}\right) .
\end{aligned}
$$

As a consequence of Theorem 2.2 is that when $\mathfrak{u} \in \mathcal{C} \ell_{Q}(V)$ is a blade, the characteristic polynomial $\chi_{\Phi_{\mathfrak{u}}}(t)$ of $\Phi_{\mathfrak{u}}$ is of the form

$$
\chi_{\Phi_{\mathfrak{u}}}(t)=\left\{\begin{array}{lll} 
\pm(t-1)^{n-\sharp \mathfrak{u}}(t+1)^{\sharp \mathfrak{u}} & \text { if } \sharp \mathfrak{u} \equiv 0 \quad(\bmod 2), \\
\pm(t-1)^{\sharp \mathfrak{u}}(t+1)^{n-\sharp \mathfrak{u}} & \text { if } \sharp \mathfrak{u} \equiv 1 & (\bmod 2) .
\end{array}\right.
$$

Recalling that (2.30) is the generating function for Kravchuk polynomials, and seeing that the characteristic polynomial of $\Phi_{\mathfrak{u}}$ is given by (2.29), (2.28) follows immediately.

Theorem 2.14 indicates that the Berezin integral is a polynomial $F(t):=$ $\int_{\mathfrak{B}} \omega d \xi_{n}(t) \cdots d \xi_{1}(t)$ having the following property:

$$
\left.\frac{d^{\ell}}{d t^{\ell}} F(t)\right|_{t=0}=\left\{\begin{array}{lll}
\ell ! K_{\ell}(n-\sharp \mathfrak{u}, n) & \text { if } \sharp \mathfrak{u} \equiv 0 & (\bmod 2), \\
\ell ! K_{\ell}(\sharp \mathfrak{u}, n) & \text { if } \sharp \mathfrak{u} \equiv 1 & (\bmod 2) .
\end{array}\right.
$$

Corollary 2.15. Let $\mathfrak{u} \in \mathcal{C} \ell_{Q}(V)$ be a blade, define $\bar{t}:=t / \mathfrak{u} \tilde{\mathfrak{u}}$, let $\left\{\mathbf{e}_{\ell}: 1 \leq \ell \leq\right.$ $n\}$ be a fixed $Q$-orthonormal basis for $V$, and for each $\ell=1, \ldots, n$, define the Clifford polynomial $v_{\ell}(t)$ by

$$
v_{\ell}(t):=\Psi_{\mathfrak{u}}\left(\mathbf{e}_{\ell}\right)-t \mathbf{e}_{\ell} .
$$

If the collection $\left\{v_{\ell}(t): 1 \leq \ell \leq n\right\}$ is orthogonal, then the corresponding iterated Berezin integral is

$$
\int_{\mathfrak{B}} \omega \mathrm{d} v_{n}(t) \cdots \mathrm{d} v_{1}(t)= \begin{cases} \pm(\mathfrak{u} \tilde{\mathfrak{u}})^{n} \sum_{\ell=0}^{n} K_{\ell}(n-\sharp \mathfrak{u}, n)(t / \mathfrak{u} \tilde{\mathfrak{u}})^{\ell} & \text { if } \sharp \mathfrak{u} \text { even, } \\ \pm(\mathfrak{u} \tilde{\mathfrak{u}})^{n} \sum_{\ell=0}^{n} K_{\ell}(\sharp \mathfrak{u}, n)(t / \mathfrak{u} \tilde{\mathfrak{u}})^{\ell} & \text { if } \sharp \mathfrak{u} \text { odd },\end{cases}
$$

where $K_{\ell}(j, n)$ denotes the $\ell^{\text {th }}$ Kravchuk polynomial of order $n$.

Proof. Noting that for each $\mathbf{x} \in V, \Psi_{\mathfrak{u}}(\mathbf{x})=(\mathfrak{u} \tilde{\mathfrak{u}}) \Phi_{\mathfrak{u}}(\mathbf{x})$, the result follows from (2.30) and the proof of Theorem 2.14.

The appearance of Kravchuk polynomials in the Berezin integral is only the first hint at the intricate relationship between Kravchuk polynomials and Clifford algebras. 


\section{OC interpretation of Kravchuk matrices}

Realizations of endomorphisms of $V$ are developed as $|V| \times|V|$ real matrices. The dual of an endomorphism $A \in \operatorname{End}(V)$, denoted $A^{\dagger}$, is realized as the matrix transpose of the right-regular representation $\check{A}$; i.e., $A^{\dagger} \mapsto \check{A}^{\dagger}$.

Given a $Q$-orthogonal basis $\left\{\mathbf{u}_{i}: 1 \leq i \leq n\right\}$ for $V$, a matrix representation of $A \in \operatorname{End}(V)$ is determined by

$$
\check{A}_{i j}=\left\langle A\left(\mathbf{u}_{i}\right), \mathbf{u}_{j}\right\rangle_{Q} .
$$

The relationships among $Q$, the $Q$-inner product, and the right-regular representation of $Q$ are understood by $Q(\mathbf{x})=\langle\mathbf{x}, \mathbf{x}\rangle_{Q}=\langle\mathbf{x}|\check{Q}| \mathbf{x}\rangle$.

Letting $\mathfrak{K}_{n}$ denote the $n^{\text {th }}$ Kravchuk matrix, which is a square matrix of order $n+1$, a number of properties are known $[9,11]$ :

- The eigenvalues of $\mathfrak{K}_{n}$ are $\lambda_{1}=2^{n / 2}$, of multiplicity $\lceil(n+1) / 2\rceil$, and $\lambda_{2}=-2^{n / 2}$, of multiplicity $\lfloor(n+1) / 2\rfloor$.

- $\mathfrak{K}_{n}{ }^{2}=2^{n} \mathcal{I}$.

- The rows of $\mathfrak{K}_{n}$ are orthogonal with respect to the order- $n$ binomial matrix $B=\left(b_{i j}\right)$ defined by

$$
b_{i j}:= \begin{cases}0 & i \neq j, \\
\left(\begin{array}{l}
n \\
j
\end{array}\right) & i=j .\end{cases}
$$

In particular, $\mathfrak{K}_{n} B \mathfrak{K}_{n}^{\dagger}=2^{n} B$.

Letting $\mathcal{K}_{n}:=2^{-n / 2} \mathfrak{K}_{n}$ denote the $n^{\text {th }}$ normalized Kravchuk matrix, the following corresponding properties are easily derived:

- The eigenvalues of $\mathcal{K}_{n}$ are $\lambda_{1}=1$, of multiplicity $\lceil(n+1) / 2\rceil$, and $\lambda_{2}=-1$, of multiplicity $\lfloor(n+1) / 2\rfloor$.

- $\mathcal{K}_{n}{ }^{2}=\mathcal{I}$.

- The rows of $\mathcal{K}_{n}$ are orthogonal with respect to the order- $n$ binomial matrix $B=\left(b_{i j}\right)$ defined by (3.2). In particular, $\mathcal{K}_{n} B \mathcal{K}_{n}^{\dagger}=B$.

In light of the results from Section 2, the first property above indicates that $\mathcal{K}_{n}$ might represent a blade conjugation operator on $\mathcal{C} \ell_{Q}(V)$. In fact, this is often the case, as the next theorem shows.

Theorem 3.1. Let $\mathcal{C} \ell_{Q}(V)$ be the Clifford algebra of a nondegenerate quadratic form $Q$ over an $(n+1)$-dimensional vector space $V$. Let $\mathcal{K}_{n}$ be the $n^{\text {th }}$ normalized Kravchuk matrix. Then,

1. When $n \equiv 0(\bmod 4), \mathcal{K}_{n}$ represents conjugation by a blade of grade $(n+$ $2) / 2$ associated with eigenspace $\mathcal{E}_{+1}$ or conjugation by a blade of grade $n / 2$ associated with eigenspace $\mathcal{E}_{-1}$. 
2. When $n \equiv 1(\bmod 4), \mathcal{K}_{n}$ represents conjugation by a blade of grade $(n+$ 1)/2 associated with eigenspace $\mathcal{E}_{+1}$.

3. When $n \equiv 3(\bmod 4), \mathcal{K}_{n}$ represents conjugation by a blade of grade $(n+$ 1)/2 associated with eigenspace $\mathcal{E}_{-1}$.

4. When $n \equiv 2(\bmod 4), \mathcal{K}_{n}$ has no consistent interpretation as a blade conjugation. However, $\mathcal{K}_{n}$ does have a consistent interpretation as generalized conjugation by a unit blade $\mathfrak{u}$ of grade $(n+2) / 2$ associated with eigenspace $\mathcal{E}_{+1}$ or generalized conjugation by a blade of grade $n / 2$ associated with eigenspace $\mathcal{E}_{-1}$, if and only if the following condition is satisfied:

$$
\mathfrak{u} \tilde{\mathfrak{u}}=\mathfrak{u}^{\star} \widetilde{\mathfrak{u}^{\star}}=-1 .
$$

Proof. Only the exceptional case, $n \equiv 2(\bmod 4)$, is treated in detail. Recall that $\mathcal{K}_{n}$ has eigenvalues $\lambda_{1}=1$ and $\lambda_{2}=-1$ of multiplicity $(n+2) / 2$ and $n / 2$, respectively. Moreover, the eigenspaces $\mathcal{E}_{+1}$ and $\mathcal{E}_{-1}$ are of dimension $(n+2) / 2$ and $n / 2$, respectively.

In the case $n \equiv 2(\bmod 4),\lceil(n+1) / 2\rceil=(n+2) / 2$ is even, thereby making -1 the eigenvalue required for consistency with blade conjugation, a contradiction. Similarly, $\lfloor(n+1) / 2\rfloor=n / 2$ is odd, making the required eigenvalue +1 , which is again a contradiction. Hence, $\mathcal{K}_{n}$ cannot represent blade conjugation.

On the other hand, if $\mathfrak{u}=\prod_{\ell=1}^{\sharp \mathfrak{u}} \mathbf{u}_{\ell}$ is a unit blade of grade $(n+2) / 2, \mathbf{x} \mid \mathfrak{u}$ implies

$$
\mathfrak{u x} \tilde{\mathfrak{u}}=(-1)^{\sharp \mathfrak{u}-1} \prod_{\ell=1}^{\sharp \mathfrak{u}} Q\left(\mathbf{u}_{\ell}\right) \mathbf{x}=(-1)^{n / 2}(\mathfrak{u} \tilde{\mathfrak{u}}) \mathbf{x} .
$$

Hence, $\mathbf{x} \mapsto \mathbf{x}$ if and only if $\mathfrak{u} \tilde{\mathfrak{u}}=-1$.

Similarly, if $\mathbf{x} \mid \mathfrak{u}^{\star}$, one finds

$$
\mathfrak{u} \mathbf{x} \tilde{\mathfrak{u}}=(-1)^{\sharp \mathfrak{u}} \prod_{\ell=1}^{\sharp \mathfrak{u}} Q\left(\mathbf{u}_{\ell}\right) \mathbf{x}=(-1)^{(n+2) / 2}(\mathfrak{u} \tilde{\mathfrak{u}}) \mathbf{x},
$$

satisfying $\mathbf{x} \mapsto-\mathbf{x}$ if and only if $\mathfrak{u} \tilde{\mathfrak{u}}=-1$. Thus, $\mathscr{K}_{n}$ represents the specified generalized conjugations in the case $n \equiv 2(\bmod 4)$.

Proofs of the remaining cases can be handled in similar fashion, once claims regarding the appropriate eigenspaces have been justified.

Observe first that the cases $n \equiv 1(\bmod 4)$ and $n \equiv 3(\bmod 4)$ lead to eigenspaces of equal dimension for $\lambda_{1}=+1$ and $\lambda_{2}=-1$. The blade conjugation requirement $\mathbf{x} \mid \mathfrak{u} \Rightarrow \mathbf{x} \mapsto(-1)^{\sharp \mathfrak{u}-1}$ thereby determines the correct eigenspace associated with the blade. Specifically, $n \equiv 1(\bmod 4)$ implies $\sharp \mathfrak{u}=(n+1) / 2$ is odd, so that the corresponding eigenvalue must be $(-1)^{\sharp u-1}=+1$. Similarly, $n \equiv 3(\bmod 4)$ implies $(n+1) / 2$ is even, so that the required eigenvalue is -1 .

In the case $n \equiv 0(\bmod 4)$ the eigenspaces are of distinct dimensions. Further, $\lceil(n+1) / 2\rceil=(n+2) / 2$ is odd, thereby making +1 the eigenvalue required 
for consistency. Similarly, $\lfloor(n+1) / 2\rfloor=n / 2$ is even, making the required eigenvalue -1 .

In light of these results, when $n \not \equiv 2(\bmod 4)$, the Kravchuk matrices now satisfy

$$
\left.\mathfrak{K}_{n} \simeq 2^{n / 2} \varphi_{\mathfrak{u}}\right|_{V}
$$

for an appropriate blade $\mathfrak{u} \in \mathcal{C} \ell_{Q}(V)$. Further, when $Q$ is positive definite, the normalized Kravchuk matrices represent compositions of reflections across orthogonal hyperplanes as detailed in Corollary 2.5.

In the case $n \equiv 2(\bmod 4)$,

$$
\left.\mathfrak{K}_{n} \simeq 2^{n / 2} \psi_{\mathfrak{u}}\right|_{V}
$$

for appropriate blade $\mathfrak{u}$ satisfying (3.3), provided $Q$ is indefinite.

Corollary 3.2. Let $\mathcal{C} \ell_{Q}(V)$ be the Clifford algebra of a nondegenerate quadratic form $Q$ over an $(n+1)$-dimensional vector space $V$. Let $\mathcal{K}_{n}$ be the $n^{\text {th }}$ normalized Kravchuk matrix. Then, $\mathcal{K}_{n}$ has the operator calculus representation

$$
\left.\mathcal{K}_{n} \simeq\left(\Lambda_{\mathfrak{u}} \Xi_{\mathfrak{u}^{-1}}+\hat{\Xi}_{\mathfrak{u}} \hat{\Lambda}_{\mathfrak{u}^{-1}}\right)\right|_{V}
$$

where

1. $\mathfrak{u}$ is a unit blade of grade $n / 2+1$ associated with eigenspace $\mathcal{E}_{+1}$, or equivalently a blade of grade $n / 2$ associated with eigenspace $\mathcal{E}_{-1}$ when $n \equiv 0$ $(\bmod 4)$;

2. $\mathfrak{u}$ is a unit blade of grade $(n+1) / 2$ associated with eigenspace $\mathcal{E}_{+1}$ when $n \equiv 1(\bmod 4)$;

3. $\mathfrak{u}$ is a unit blade of grade $(n+1) / 2$ associated with eigenspace $\mathcal{E}_{-1}$ when $n \equiv 3(\bmod 4)$.

When $n \equiv 2(\bmod 4), \mathcal{K}_{n}$ has the operator calculus representation

$$
\left.\mathcal{K}_{n} \simeq\left(\Lambda_{\mathfrak{u}} \Xi_{\tilde{u}}+\hat{\Xi}_{\mathfrak{u}} \hat{\Lambda}_{\tilde{u}}\right)\right|_{V}
$$

where $\mathfrak{u}$ is a blade of grade $(n+2) / 2$ associated with eigenspace $\mathcal{E}_{+1}$ or a blade of grade $n / 2$ associated with eigenspace $\mathcal{E}_{-1}$, provided that the following condition is satisfied:

$$
\mathfrak{u} \tilde{\mathfrak{u}}=\mathfrak{u}^{\star} \widetilde{\mathfrak{u}^{\star}}=-1 .
$$


Proof. By Lemma 2.1, the conjugation operator satisfies $\varphi_{\mathfrak{u}}=\Lambda_{\mathfrak{u}} \Xi_{\mathfrak{u}^{-1}}+\hat{\Xi}_{\mathfrak{u}} \hat{\Lambda}_{\mathfrak{u}^{-1}}$. Similarly, (1.31), (1.32), and Lemma 1.5 imply

$$
\begin{aligned}
& \psi_{\mathfrak{u}}=\left(\Lambda_{\mathfrak{u}} \oplus \hat{\Xi}_{\mathfrak{u}}\right)\left(\hat{\Lambda}_{\tilde{\mathfrak{u}}} \oplus \Xi_{\tilde{\mathfrak{u}}}\right) \\
& =\Lambda_{\mathfrak{u}} \hat{\Lambda}_{\tilde{u}}+\hat{\Xi}_{\mathfrak{u}} \Xi_{\tilde{u}}+\Lambda_{\mathfrak{u}} \Xi_{\tilde{u}}+\hat{\Xi}_{\mathfrak{u}} \hat{\Lambda}_{\tilde{u}} \\
& =\Lambda_{\mathfrak{u}} \Xi_{\tilde{u}}+\hat{\Xi}_{\mathfrak{u}} \hat{\Lambda}_{\tilde{u}}
\end{aligned}
$$

for generalized conjugation as in (3.9). The rest follows from Theorem 3.1.

To better see that the case $n \equiv 2(\bmod 4)$ is not vacuous, i.e., that $(3.3)$ has solutions, consider the following. First, let $\mathfrak{S}$ denote an $(n+1) \times(n+1)$ diagonal matrix with $\{ \pm 1\}$ along the diagonal such that among the first $n / 2+1$ diagonal entries, -1 appears an odd number of times. Similarly, -1 should appear an odd number of times among the remaining $n / 2$ diagonal entries.

Let $E$ be a matrix whose rows are unit eigenvectors of $\mathcal{K}_{n}$ and set $Q_{\kappa}:=$ $E^{-1} \mathfrak{S} E^{\dagger^{-1}}$, noting that $Q_{\kappa}$ is then nonsingular and symmetric. It follows that $Q_{k}$ represents a nondegenerate quadratic form satisfying

$$
E Q_{\kappa} E^{\dagger}=\mathfrak{S} \text {. }
$$

Denoting the $i^{\text {th }}$ row of $E$ by $k_{i}$, define the blade $\mathfrak{u}:=k_{1} \cdots k_{(n / 2)+1} \in \mathcal{C} \ell_{Q_{\kappa}}(V)$. It follows that $\mathfrak{u}^{\star}=k_{(n / 2)+2} \cdots k_{n}$.

By construction, it now follows that $\mathfrak{u} \tilde{\mathfrak{u}}=\mathfrak{u}^{\star} \tilde{\mathfrak{u}}^{\star}=-1$, satisfying (3.3). Moreover, one can see that

$$
\mathfrak{u} \mathbf{x} \tilde{\mathfrak{u}}= \begin{cases}\mathbf{x}=(\mathfrak{u} \tilde{\mathfrak{u}})(-1)^{(n / 2+1)-1} \mathbf{x} & \text { if } \mathbf{x} \mid \mathfrak{u}, \\ -\mathbf{x}=(\mathfrak{u} \tilde{\mathfrak{u}})(-1)^{(n / 2+1)} \mathbf{x} & \text { if } \mathbf{x} \mid \mathfrak{u}^{\star}\end{cases}
$$

so that $\mathbf{x} \mapsto \mathfrak{u x} \tilde{\mathfrak{u}}$ represents generalized conjugation by blade $\mathfrak{u}$, in accordance with (2.13). Similarly, one establishes that $\mathscr{K}_{n}$ also represents generalized conjugation by $\mathfrak{u}^{\star}$.

\section{Spectral Properties of Induced Operators}

The notation $X \rightarrow \mathfrak{X}$ will be used to indicate that an operator $\mathfrak{X}$ on $\mathcal{C} \ell_{Q}(V)$ has been induced by the action of operator $X$ on the underlying vector space, $V$.

Definition 4.1. An operator $X \in \mathcal{L}(V)$ is said to be deduced from $\mathfrak{X} \in$ $\mathcal{L}\left(\mathcal{C} \ell_{Q}(V)\right)$ if $X \rightarrow \mathfrak{X}$. In this case, it is clear that $X=\left.\mathfrak{X}\right|_{V}$.

The grade function gr: $\mathcal{C} \ell_{Q}(V) \rightarrow \mathbb{N}_{0}$ is defined on an arbitrary multivector $u=\sum_{I \in 2^{[n]}} \alpha_{I} \mathfrak{u}_{I} \in \mathcal{C} \ell_{Q}(V)$ by

$$
\operatorname{gr}(u):=\max _{\left\{I \in 2^{[n]}: \alpha_{I} \neq 0\right\}} \sharp \mathfrak{u} .
$$

It should be clear that the grade function is basis independent. 
Definition 4.2. An operator $\varphi$ on $\mathcal{C} \ell_{Q}(V)$ satisfying $\operatorname{gr}(\varphi(u)) \leq \operatorname{gr}(u)$ for all blades $u \in \mathcal{C} \ell_{Q}(V)$ is said to be lowering. Similarly, if $\operatorname{gr}(\varphi(u)) \geq \operatorname{gr}(u)$ for all blades $u \in \mathcal{C} \ell_{Q}(V)$, then $\varphi$ is said to be raising. The operator $\varphi$ is said to be grade preserving if it is both lowering and raising.

As a consequence of $Q$-orthogonality, $\varphi_{\mathfrak{u}}$ is blade preserving; i.e., the image of a blade is a blade. Hence, the level- $\ell$ induced map $\varphi_{\mathfrak{u}}{ }^{(\ell)}$ is a well-defined linear operator on $\bigwedge^{\ell} V$, the grade- $\ell$ subspace of $\mathcal{C} \ell_{Q}(V)$ spanned by the basis $\ell$-vectors.

The first result shows that, as in the case of the level-1 map $\Phi_{\mathfrak{u}}$, the level- $\ell$ map determines a quantum observable.

Lemma 4.3. The level- $\ell$ induced map $\varphi_{\mathfrak{u}}{ }^{(\ell)}$ is self-adjoint with respect to $\langle\cdot, \cdot\rangle_{Q}$. Moreover, $\varphi_{\mathfrak{u}}{ }^{(\ell)}$ is Q-orthogonal.

Proof. Letting $\mathfrak{x}$ and $\mathfrak{y}$ be arbitrary $\ell$-blades in $\mathcal{C} \ell_{Q}(V)$, it suffices to show that $\left\langle\varphi_{\mathfrak{u}}{ }^{(\ell)}(\mathfrak{x}), \mathfrak{y}\right\rangle_{Q}=\left\langle\mathfrak{x}, \varphi_{\mathfrak{u}}{ }^{(\ell)}(\mathfrak{y})\right\rangle_{Q}$.

First, since $\mathfrak{u}$ is a blade, let $\mathfrak{U}=\left\{\mathbf{u}_{1}, \ldots, \mathbf{u}_{n}\right\}$ be a $Q$-orthogonal basis for $V$ such that $\mathfrak{u}$ is a basis blade for the grade $\sharp \mathfrak{u}$ subspace of $\mathcal{C} \ell_{Q}(V)$. Hence, one can write $\mathfrak{u}=\mathbf{u}_{1} \cdots \mathbf{u}_{\sharp \mathfrak{u}}$. Further, $\mathfrak{x}$ and $\mathfrak{y}$ can be written as linear combinations of basis $\ell$-blades: $\mathfrak{x}=\sum_{|J|=\ell} \alpha_{J} \mathbf{u}_{J}$ and $\mathfrak{y}=\sum_{|I|=\ell} \beta_{I} \mathbf{u}_{I}$. It is therefore sufficient to show that $\left\langle\varphi_{\mathbf{u}}^{(\ell)}\left(\mathbf{u}_{I}\right), \mathbf{u}_{J}\right\rangle_{Q}=\left\langle\mathbf{u}_{I}, \varphi_{\mathfrak{u}}{ }^{(\ell)}\left(\mathbf{u}_{J}\right)\right\rangle_{Q}$ holds for arbitrary basis $\ell$-blades $\mathbf{u}_{I}$ and $\mathbf{u}_{J}$.

Since each vector of $\mathbf{u}_{I}$ is either a factor of $\mathfrak{u}$ or of its orthogonal complement $\mathfrak{u}^{\star}, \mathfrak{u} \mathbf{u}_{I} \mathfrak{u}^{-1}=\prod_{i \in I} \mathfrak{u} \mathbf{u}_{i} \mathfrak{u}^{-1}$ implies

$$
\begin{aligned}
\mathfrak{u}_{\mathbf{u}} \mathfrak{u}^{-1} \widetilde{\mathbf{u}_{J}}= & \left(\prod_{i \in I: 1 \leq i \leq \sharp \mathfrak{u}}(-1)^{\sharp \mathfrak{u}-1} \prod_{i \in I: i>\sharp \mathfrak{u}}(-1)^{\sharp \mathfrak{u}} \mathbf{u}_{I}\right) \widetilde{\mathbf{u}_{J}} \\
= & \left(\prod_{i \in I: 1 \leq i \leq \sharp \mathfrak{u}}(-1)^{\sharp \mathfrak{u}-1} \prod_{i \in I: i>\sharp \mathfrak{u}}(-1)^{\sharp \mathfrak{u}} \mathbf{u}_{I}\right)(-1)^{\frac{\ell(\ell-1)}{2}} \mathbf{u}_{J} \\
& =(-1)^{\frac{\ell(\ell-1)}{2}}\left(\prod_{i \in I: 1 \leq i \leq \sharp \mathfrak{u}}(-1)^{\sharp \mathfrak{u}-1} \prod_{i \in I: i>\sharp \mathfrak{u}}(-1)^{\sharp \mathfrak{u}}\right) \mathbf{u}_{I} \mathbf{u}_{J} .
\end{aligned}
$$

On the other hand, a similar calculation shows

$$
\widetilde{\mathbf{u}_{I} \mathfrak{u} \mathbf{u}_{J} \mathfrak{u}^{-1}}=(-1)^{\frac{\ell(\ell-1)}{2}}\left(\prod_{j \in J: 1 \leq j \leq \sharp \mathfrak{u}}(-1)^{\sharp \mathfrak{u}-1} \prod_{j \in J: j>\sharp \mathfrak{u}}(-1)^{\sharp \mathfrak{u}}\right) \mathbf{u}_{I} \mathbf{u}_{J} .
$$

Note that the product $\mathbf{u}_{I} \mathbf{u}_{J}$ is given by

$$
\mathbf{u}_{I} \mathbf{u}_{J}=(-1)^{\sum_{j \in J}|\{i \in I: i>j\}|} \prod_{k \in I \cap J} Q\left(\mathbf{u}_{k}\right) \mathbf{u}_{I \triangle J},
$$


where $I \triangle J$ is the set-symmetric difference of $I$ and $J$. Hence,

$$
\left\langle\mathbf{u}_{I} \mathbf{u}_{J}\right\rangle_{0}= \begin{cases}(-1)^{\sum_{j \in J}|\{i \in I: i>j\}|} \prod_{k \in I \cap J} Q\left(\mathbf{u}_{k}\right) & \text { if } I=J, \\ 0 & \text { otherwise }\end{cases}
$$

It now follows that $\mathfrak{u} \mathbf{u}_{I} \mathfrak{u}^{-1} \widetilde{\mathbf{u}_{J}}$ is nonzero only if $I=J$. Note that $I=J$ implies

$$
\begin{aligned}
(-1)^{\frac{\ell(\ell-1)}{2}}\left(\prod_{i \in I: 1 \leq i \leq \sharp \mathfrak{u}}(-1)^{\sharp \mathfrak{u}-1} \prod_{i \in I: i>\sharp \mathfrak{u}}(-1)^{\sharp \mathfrak{u}}\right) \\
=(-1)^{\frac{\ell(\ell-1)}{2}}\left(\prod_{j \in J: 1 \leq j \leq \sharp \mathfrak{u}}(-1)^{\sharp \mathfrak{u}-1} \prod_{j \in J: j>\sharp \mathfrak{u}}(-1)^{\sharp \mathfrak{u}}\right),
\end{aligned}
$$

which shows (4.2) is equal to (4.3).

Orthogonality is similarly established:

$$
\begin{array}{r}
\left\langle\mathfrak{u} \mathbf{u}_{I} \mathfrak{u}^{-1}, \mathfrak{u} \mathbf{u}_{J} \mathfrak{u}^{-1}\right\rangle_{Q}=\left\langle\mathfrak{u} \mathbf{u}_{I} \mathfrak{u}^{-1} \widetilde{\mathfrak{u} \mathbf{u}_{J} \mathfrak{u}^{-1}}\right\rangle_{0}=(-1)^{\frac{\ell(\ell-1)}{2}}\left\langle\mathfrak{u} \mathbf{u}_{I} \mathfrak{u}^{-1} \mathfrak{u u}_{J} \mathfrak{u}^{-1}\right\rangle_{0} \\
=\left\langle(-1)^{\frac{\ell(\ell-1)}{2}}\left(\prod_{j \in J: 1 \leq j \leq \sharp \mathfrak{u}}(-1)^{\sharp \mathfrak{u}-1} \prod_{j \in J: j>\sharp \mathfrak{u}}(-1)^{\sharp \mathfrak{u}}\right)^{2} \mathbf{u}_{I} \mathbf{u}_{J}\right\rangle_{0} \\
=\left\langle(-1)^{\frac{\ell(\ell-1)}{2}} \mathbf{u}_{I} \mathbf{u}_{J}\right\rangle_{0}=\left\langle\mathbf{u}_{I} \widetilde{\mathbf{u}_{J}}\right\rangle_{0}=\left\langle\mathbf{u}_{I}, \mathbf{u}_{J}\right\rangle_{Q} .
\end{array}
$$

Hence, $\varphi_{\mathfrak{u}}{ }^{(\ell)}$ is a quantum observable for each $\ell=1, \ldots, n$. The expectation of this quantum observable is given by its trace.

Lemma 4.4. For fixed blade $\mathfrak{u} \in \mathcal{C} \ell_{Q}(V)$, the level- $\ell$ induced map $\varphi_{\mathfrak{u}}{ }^{(\ell)}$ has eigenvalues \pm 1 . The dimensions of the corresponding eigenspaces are given by

$$
\operatorname{dim}\left(\mathcal{E}_{-1}{ }^{(\ell)}\right)= \begin{cases}\sum_{j \text { odd }}\left(\begin{array}{c}
\sharp \mathfrak{u} \\
j
\end{array}\right)\left(\begin{array}{c}
n-\sharp \mathfrak{u} \\
\ell-j
\end{array}\right) & \sharp \mathfrak{u} \equiv 0 \quad(\bmod 2), \\
\sum_{j \text { odd }}\left(\begin{array}{c}
n-\sharp \mathfrak{u} \\
j
\end{array}\right)\left(\begin{array}{c}
\sharp \mathfrak{u} \\
\ell-j
\end{array}\right) & \sharp \mathfrak{u} \equiv 1 \quad(\bmod 2),\end{cases}
$$

and

$$
\operatorname{dim}\left(\mathcal{E}_{+1}{ }^{(\ell)}\right)=\left\{\begin{array}{l}
\sum_{j \text { even }}\left(\begin{array}{c}
\sharp \mathfrak{u} \\
j
\end{array}\right)\left(\begin{array}{c}
n-\sharp \mathfrak{u} \\
\ell-j
\end{array}\right) \\
\sum_{j \text { even }}\left(\begin{array}{c}
n-\sharp \mathfrak{u} \\
j
\end{array}\right)\left(\begin{array}{c}
\sharp \mathfrak{u} \\
\ell-j
\end{array}\right) \quad \sharp \mathfrak{u} \equiv 1 \quad(\bmod 2),
\end{array}\right.
$$

with sums being taken over all admissible values of the index. 
Proof. Let $\mathfrak{U}_{1}, \mathfrak{U}_{2}$ be $Q$-orthogonal unit bases of the $\varphi_{\mathfrak{u}}$ eigenspaces $\mathcal{E}_{\lambda_{1}}$ and $\mathcal{E}_{\lambda_{2}}$, respectively. Writing $\mathfrak{U}_{1}=\left\{\mathbf{u}_{1}, \ldots, \mathbf{u}_{\sharp \mathfrak{u}}\right\}$ and $\mathfrak{U}_{2}=\left\{\mathbf{u}_{\sharp \mathfrak{u}+1}, \ldots, \mathbf{u}_{n}\right\}$, one immediately sees that

$$
\varphi_{\mathfrak{u}}\left(\mathbf{u}_{I}\right)=\prod_{\left|I \cap \mathfrak{U}_{1}\right|}(-1)^{\sharp \mathfrak{u}-1} \prod_{\left|I \cap \mathfrak{U}_{2}\right|}(-1)^{\sharp \mathfrak{u}} \mathbf{u}_{I} .
$$

Computing the dimension of $\mathcal{E}_{-1}{ }^{(\ell)}$ thereby amounts to counting the multiindices $I \in 2^{[n]}$ in which an odd number of generators having negative eigenvalues occur. When $\sharp \mathfrak{u}$ is even, an odd number of generators in $I$ must come from $\mathfrak{U}_{1}$ and an even number from $\mathfrak{U}_{2}$. When $\sharp \mathfrak{u}$ is odd, an even number of generators must come from $\mathfrak{U}_{1}$ and an odd number from $\mathfrak{U}_{2}$. Keeping in mind that $\left|\mathfrak{U}_{1}\right|=\sharp \mathfrak{u}$ and $\left|\mathfrak{U}_{2}\right|=n-\sharp \mathfrak{u},(4.8)$ is established by counting cases. The dimension of $\mathcal{E}_{+1}{ }^{(\ell)}$ is computed by similar counting arguments to reveal (4.9).

Corollary 4.5. The trace and determinant of the level- $\ell$ induced operator are given by the following:

$$
\operatorname{tr}\left(\varphi_{\mathfrak{u}}{ }^{(\ell)}\right)=\left\{\begin{array}{lll}
K_{\ell}(\sharp \mathfrak{u}, n) & \sharp \mathfrak{u} \equiv 0 & (\bmod 2), \\
K_{\ell}(n-\sharp \mathfrak{u}, n) & \sharp \mathfrak{u} \equiv 1 & (\bmod 2),
\end{array}\right.
$$

and

$$
\operatorname{det}\left(\varphi_{\mathfrak{u}}{ }^{(\ell)}\right)=(-1)^{\operatorname{dim}\left(\mathcal{E}_{-1}^{(\ell)}\right)} .
$$

Proof. The trace follows from the explicit formula for the $\ell^{\text {th }}$ Kravchuk polynomial of order $n$ seen in (1.2). The determinant follows from Lemma 4.4.

Recall that the level- $\ell$ induced map is the restriction of $\varphi_{\mathfrak{u}}$ to the grade- $\ell$ subspace of $\mathcal{C} \ell_{Q}(V)$; i.e.,for each $\ell=0, \ldots, n$,

$$
\varphi_{\mathfrak{u}}^{(\ell)}=\left.\varphi_{\mathfrak{u}}\right|_{\wedge^{\ell} V}
$$

Further observing that these subspaces are $Q$-orthogonal to each other, $\varphi_{\mathfrak{u}}$ is seen to be a direct operator sum:

$$
\varphi_{\mathfrak{u}}:=\bigoplus_{\ell=0}^{n} \varphi_{\mathfrak{u}}{ }^{(\ell)}
$$

Relative to an ordered $Q$-orthogonal basis $\left\{\mathbf{u}_{j}: 1 \leq j \leq n\right\}$ of $V$ and induced canonical blade basis $\left\{\mathbf{u}_{I}: I \in 2^{[n]}\right\}$, the matrix representation of the level- $\ell$ induced map of $\varphi_{\mathfrak{u}}$ is an order $\left(\begin{array}{l}n \\ \ell\end{array}\right)$ square matrix.

For integer $i \in\left\{0, \ldots, 2^{n}-1\right\}$, let $\underline{i}$ denote the corresponding subset representation of $i$; that is, $\underline{i}$ is a subset of the $n$-set, $[n]$, satisfying $i=\sum_{\ell \in \underline{i}} 2^{\ell}$. An ordering is then naturally imposed on the basis blades by

$$
\mathbf{u}_{\underline{i}} \prec \mathbf{u}_{\underline{j}}\left\{\begin{array}{l}
\text { if }|\underline{i}|<|\underline{j}|, \text { or } \\
\text { if }|\underline{i}|=|\underline{j}| \text { and } i<j .
\end{array}\right.
$$


The matrix representation of $\varphi_{\mathfrak{u}}$ with respect to the basis ordered by (4.15) is then a $2^{n} \times 2^{n}$ block-diagonal matrix $\check{\varphi}_{\mathfrak{u}}=\left(\phi_{I J}\right)$ defined by

$$
\phi_{I J}:=\left\langle\mathbf{u}_{I}\left|\varphi_{\mathfrak{u}}\right| \mathbf{u}_{J}\right\rangle
$$

for multi-indices $I, J \in 2^{[n]}$.

For integers $k, \ell$ and matrix $M$, the notation $\langle k|M| \ell\rangle$ will denote the entry of $M$ in row $k$, column $\ell$. Letting $\langle\mathbf{1}|$ denote the row vector of ones allows the $j^{\text {th }}$ column sum to be written $\langle\mathbf{1}|M| j\rangle$.

Corollary 4.6. The trace of the blade conjugation operator on $\mathcal{C} \ell_{Q}(V)$ is obtained by summing a column of the $n^{\text {th }}$ Kravchuk matrix, $\mathfrak{K}_{n}$. More specifically, letting 1 denote the vector of all ones,

$$
\operatorname{tr}\left(\varphi_{\mathfrak{u}}\right)=\left\{\begin{array}{lll}
\sum_{\ell=0}^{n} K_{\ell}(\sharp \mathfrak{u}, n)=\left\langle\mathbf{1}\left|\mathfrak{K}_{n}\right| \sharp \mathfrak{u}\right\rangle & \text { if } \sharp \mathfrak{u} \equiv 0 & (\bmod 2), \\
\sum_{\ell=0}^{n} K_{\ell}(n-\sharp \mathfrak{u}, n)=\left\langle\mathbf{1}\left|\mathfrak{K}_{n}\right| n-\sharp \mathfrak{u}\right\rangle & \text { if } \sharp \mathfrak{u} \equiv 1 \quad(\bmod 2) .
\end{array}\right.
$$

Proof. Since $\varphi_{\mathfrak{u}}$ is blade-preserving, its matrix is block-diagonal (under appropriate ordering of basis). The rest follows from summing over levels.

Given an orthogonal collection $\mathfrak{U}=\left\{\mathbf{u}_{j}: 1 \leq j \leq|\mathfrak{U}|\right\}$ of blades in $\mathcal{C} \ell_{Q}(V)$, attention now turns to linear combinations of operators $\xi=\sum_{j=1}^{|\mathfrak{U}|} a_{j} \varphi_{\mathfrak{u}_{j}}$. Since $\varphi_{\mathfrak{u}}$ is self-adjoint for each $\mathfrak{u}$, bilinearity of $\langle\cdot, \cdot\rangle_{Q}$ guarantees that $\xi$ is self-adjoint with respect to the inner product $\langle\cdot, \cdot\rangle_{Q}$. Hence, $\xi$ also represents a quantum random variable.

Further, the restriction of $\xi$ to the grade- $\ell$ subspace $\bigwedge^{\ell} V$ is once again a well-defined linear operator. The level- $\ell$ induced operator is defined accordingly:

$$
\xi^{(\ell)}:=\left.\xi\right|_{\Lambda^{\ell} V} .
$$

The next result concerns traces of linear combinations of conjugation operators of a specified grade. It is here that the symmetric Kravchuk matrices $\mathfrak{K}_{n}{ }^{\text {sym }}$ come into play.

Proposition 4.7. Let $\mathfrak{U}$ denote a fixed orthogonal basis of unit blades for $\mathcal{C} \ell_{Q}(V)$. For fixed $g \in\{0,1, \ldots, n\}$, define the operator $\xi_{g}:=\sum_{\{\mathfrak{u} \in \mathfrak{U}: \sharp \mathfrak{u}=g\}} a_{\mathfrak{u}} \varphi_{\mathfrak{u}}$, for scalar coefficients $a_{\mathfrak{u}}$. Then,

$$
\operatorname{tr}\left(\xi_{g}{ }^{(\ell)}\right)=\left\{\begin{array}{lll}
K_{\ell}(g, n) \sum_{\mathfrak{u} \in \mathfrak{U}} a_{\mathfrak{u}} & \text { if } g \equiv 0 & (\bmod 2), \\
K_{\ell}(n-g, n) \sum_{\mathfrak{u} \in \mathfrak{U}} a_{\mathfrak{u}} & \text { if } g \equiv 1 & (\bmod 2) .
\end{array}\right.
$$


In particular, when $a_{\mathfrak{u}}=1$ for all $\mathfrak{u}$,

$$
\operatorname{tr}\left(\xi_{g}\right)=\left\{\begin{array}{lll}
\left\langle\mathbf{1}\left|\mathfrak{K}_{n}{ }^{\text {sym }}\right| g\right\rangle & \text { if } g \equiv 0 & (\bmod 2), \\
\left\langle\mathbf{1}\left|\mathfrak{K}_{n}{ }^{\mathrm{sym}}\right| n-g\right\rangle & \text { if } g \equiv 1 & (\bmod 2) .
\end{array}\right.
$$

In other words, the trace of $\xi_{g}$ is obtained by summing the appropriate column of the symmetric Kravchuk matrix $\mathfrak{K}_{n}{ }^{\mathrm{sym}}$.

Proof. Linearity of the trace functional together with Corollary 4.6 establishes (4.19). If $a_{\mathfrak{u}}=1$ for all $\mathfrak{u}$, these sums of coefficients are reduced to binomial coefficients:

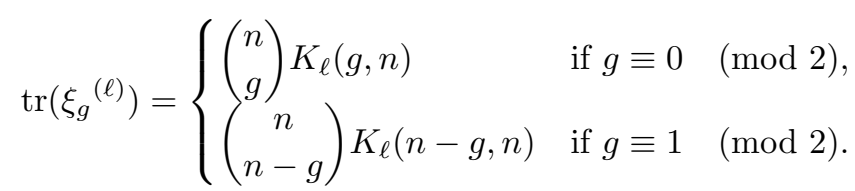

Summing over levels then gives the sum of the $g^{\text {th }}$ column of the symmetric Kravchuk matrix $\mathfrak{K}_{n}{ }^{\mathrm{sym}}$.

\section{$5 \quad$ Reductions and Other Inductions}

As already seen, a linear operator $A$ on an $n$-dimensional vector space naturally induces an operator on the $2^{n}$-dimensional Clifford algebra $\mathcal{C} \ell_{Q}(V)$. Dual to this notion is the idea of reducing an operator on $\mathcal{C} \ell_{Q}(V)$ to an operator on the paravectors of $V$, that is, $\mathbb{R} \oplus V$, which we denote by $V_{*}$.

Definition 5.1. Let $\mathfrak{A}$ be an endomorphism on $\mathcal{C} \ell_{Q}(V)$, defined by its action on basis blades. An operator $A$ on $V_{*}$ is said to be grade-reduced from $\mathfrak{A}$ if its action on the ordered generators $\left\{\varepsilon_{0}, \ldots, \varepsilon_{n}\right\}$ of $V_{*}$ satisfies

$$
\left\langle\varepsilon_{i}|A| \varepsilon_{j}\right\rangle:=\sum_{\substack{\sharp \mathfrak{a}=i \\ \sharp \mathfrak{b}=j}}\langle\mathfrak{a}|\mathfrak{A}| \mathfrak{b}\rangle,
$$

where the sum is taken over blades in some fixed basis of $\mathcal{C} \ell_{Q}(V)$.

When (5.1) holds, it is convenient to write $\mathfrak{A} \searrow A$ and refer to $A$ as an operator grade-reduced from $\mathfrak{A}$. Similarly, if $X$ is a linear operator on $V_{*}$ having ordered basis $\left\{\varepsilon_{0}, \varepsilon_{1}, \ldots, \varepsilon_{n}\right\}$, the operator $\mathfrak{X}$ on $\mathcal{C} \ell_{Q}(V)$ whose action is defined on basis blades by

$$
\mathfrak{X}(\mathfrak{u}):=\sum_{\mathfrak{v} \in \mathcal{B}}\left\langle\varepsilon_{\sharp \mathfrak{u}}|X| \varepsilon_{\sharp \mathfrak{v}}\right\rangle \mathfrak{v}
$$

is said to be grade-induced on $\mathcal{C} \ell_{Q}(V)$ by $X$. When (5.2) holds, it is convenient to write $X \nearrow \mathfrak{X}$. 
Considering spaces of linear operators, one sees

$$
\mathcal{L}\left(V_{*}\right) \nearrow \mathcal{L}\left(\mathcal{C} \ell_{Q}(V)\right) \searrow \mathcal{L}\left(V_{*}\right)
$$

More specifically, one has the next sequence of lemmas and corollaries.

Lemma 5.2. Regarding the diagonal matrix $B$ of binomial coefficients as an operator on $V_{*}$, let $X \in \mathcal{L}\left(V_{*}\right)$. Then, $X \nearrow \mathfrak{X} \searrow B X B$.

Proof. Suppose $X \nearrow \mathfrak{X}$. Then, letting $A$ denote the grade reduction of $\mathfrak{X}$,

$$
\left\langle\varepsilon_{i}|A| \varepsilon_{j}\right\rangle:=\sum_{\substack{\sharp \mathfrak{a}=i \\
\sharp \mathfrak{b}=j}}\langle\mathfrak{a}|\mathfrak{X}| \mathfrak{b}\rangle=\sum_{\substack{\sharp \mathfrak{a}=i \\
\sharp \mathfrak{b}=j}}\left\langle\varepsilon_{\sharp \mathfrak{a}}|X| \varepsilon_{\sharp \mathfrak{b}}\right\rangle=\left(\begin{array}{c}
n \\
i
\end{array}\right)\left\langle\varepsilon_{i}|X| \varepsilon_{j}\right\rangle\left(\begin{array}{c}
n \\
j
\end{array}\right) .
$$

For convenience, the notation $A \rightarrow \mathfrak{A}$ will be used to indicate that the operator $\mathfrak{A}$ on $\mathcal{C} \ell_{Q}(V)$ is induced by the (conjugation) action of $A$ on $V$. Considering spaces of linear operators on $V$, its Clifford algebra $\mathcal{C} \ell_{Q}(V)$, and the space of paravectors $V_{*}:=\mathbb{R} \oplus V$, induced and reduced operators satisfy the following:

$$
\mathcal{L}(V) \rightarrow \mathcal{L}\left(\mathcal{C} \ell_{Q}(V)\right) \searrow \mathcal{L}\left(V_{*}\right) .
$$

Lemma 5.3. Let $X \in \mathcal{L}\left(V_{*}\right)$, suppose $X \nearrow \mathfrak{X}$, and let $B$ be the diagonal matrix of binomial coefficients regarded as an operator on $V_{*}$. Then,

$$
\operatorname{tr}(\mathfrak{X})=\operatorname{tr}(X B) .
$$

Proof. Suppose $X \nearrow \mathfrak{X}$. Then,

$$
\begin{aligned}
\operatorname{tr}(\mathfrak{X})=\sum_{\mathfrak{a} \in \mathfrak{B}}\langle\mathfrak{a}|\mathfrak{X}| \mathfrak{a}\rangle & =\sum_{i=0}^{n} \sum_{\sharp \mathfrak{a}=i}\langle\mathfrak{a}|\mathfrak{X}| \mathfrak{a}\rangle \\
& =\sum_{i=0}^{n} \sum_{\sharp \mathfrak{a}=i}\left\langle\varepsilon_{i}|X| \varepsilon_{i}\right\rangle=\sum_{i=0}^{n}\left(\begin{array}{c}
n \\
i
\end{array}\right)\left\langle\varepsilon_{i}|X| \varepsilon_{i}\right\rangle=\operatorname{tr}(X B) .
\end{aligned}
$$

Lemma 5.4. Let $X, Y \in \mathcal{L}\left(V_{*}\right)$, suppose $X \nearrow \mathfrak{X}$ and $Y \nearrow \mathfrak{Y}$, and let $\mathfrak{X} \circ \mathfrak{Y}$ denote the operator on $\mathcal{C} \ell_{Q}(V)$ represented by the Hadamard product of matrix representations of the grade-induced operators. Then,

$$
\operatorname{tr}(\mathfrak{X} \circ \mathfrak{Y})=\operatorname{tr}((X \circ Y) B),
$$

where $B$ is the diagonal matrix of binomial coefficients regarded as an operator on $V_{*}$. 
Proof. First, observe that

$$
\begin{aligned}
(\mathfrak{X} \circ \mathfrak{Y})(\mathfrak{u}) & =\sum_{\mathfrak{v} \in \mathfrak{B}}\langle\mathfrak{u}|\mathfrak{X} \circ \mathfrak{Y}| \mathfrak{v}\rangle \mathfrak{v} \\
= & \sum_{\mathfrak{v} \in \mathfrak{B}}\langle\mathfrak{u}|\mathfrak{X}| \mathfrak{v}\rangle\langle\mathfrak{u}|\mathfrak{Y}| \mathfrak{v}\rangle \mathfrak{v}=\sum_{\mathfrak{v} \in \mathfrak{B}}\left\langle\varepsilon_{\sharp \mathfrak{u}}|X| \varepsilon_{\sharp \mathfrak{v}}\right\rangle\left\langle\varepsilon_{\sharp \mathfrak{u}}|Y| \varepsilon_{\sharp \mathfrak{v}}\right\rangle \mathfrak{v} \\
& =\sum_{\mathfrak{v} \in \mathfrak{B}}\left\langle\varepsilon_{\sharp \mathfrak{u}}|X \circ Y| \varepsilon_{\sharp \mathfrak{v}}\right\rangle \mathfrak{v} .
\end{aligned}
$$

Hence,

$$
\begin{aligned}
\operatorname{tr}(\mathfrak{X} \circ \mathfrak{Y})= & \sum_{\mathfrak{u} \in \mathfrak{B}} \sum_{\mathfrak{v} \in \mathfrak{B}}\langle\mathfrak{u}|\mathfrak{X} \circ \mathfrak{Y}| \mathfrak{u}\rangle \\
& =\sum_{\mathfrak{v} \in \mathfrak{B}}\langle\mathfrak{u}|\mathfrak{X}| \mathfrak{v}\rangle\langle\mathfrak{u}|\mathfrak{Y}| \mathfrak{v}\rangle \mathfrak{v}=\sum_{\mathfrak{u} \in \mathfrak{B}}\left\langle\varepsilon_{\sharp \mathfrak{u}}|X| \varepsilon_{\sharp \mathfrak{u}}\right\rangle\left\langle\varepsilon_{\sharp \mathfrak{u}}|Y| \varepsilon_{\sharp \mathfrak{u}}\right\rangle \\
& =\sum_{\ell=0}^{n}\left(\begin{array}{c}
n \\
\ell
\end{array}\right)\left\langle\varepsilon_{\ell}|X \circ Y| \varepsilon_{\ell}\right\rangle=\operatorname{tr}((X \circ Y) B) .
\end{aligned}
$$

Proposition 5.5. Regarding the Kravchuk matrix, $\mathfrak{K}_{n}$, and the symmetric Kravchuk matrix, $\mathfrak{K}_{n}{ }^{\mathrm{sym}}$, as operators on $V_{*}$ and letting $\mathcal{K}$ denote the appropriate grade induced operator on $\mathcal{C} \ell_{Q}(V)$, one immediately sees

$$
\mathfrak{K}_{n} \nearrow \mathcal{K} \searrow B \mathfrak{K}_{n}{ }^{\mathrm{sym}} \text {. }
$$

Proof. The result follows immediately from Lemma 5.2 by observing $B \mathfrak{K}_{n} B=$ $B\left(\mathfrak{K}_{n} B\right)=B \mathfrak{K}_{n}{ }^{\mathrm{sym}}$.

Remark 5.6. In fact, letting $\mathfrak{B}$ denote the operator on $\mathcal{C} \ell_{Q}(V)$ grade-induced by $B^{-1}$ on $V_{*}$, one finds $B^{-1} \nearrow \mathfrak{B} \searrow B$. Moreover,

$$
B^{-2} \nearrow \mathfrak{B}^{\circ 2} \searrow \mathcal{I}
$$

where $\mathfrak{B}^{\circ 2}=\mathfrak{B} \circ \mathfrak{B}$ denotes the operator on $\mathcal{C} \ell_{Q}(V)$ represented by the Hadamard product of matrix representations of $\mathfrak{B}$.

Proposition 5.7. If $\Psi$ is $Q$-orthogonal on $V$, then there exists a diagonal operator $\Delta$ on $V_{*}$ satisfying

$$
\Psi \rightarrow \psi \searrow \Delta .
$$

Proof. If $\Psi$ is $Q$-orthogonal on $V$, then the induced operator $\psi$ is grade-preserving on $\mathcal{C} \ell_{Q}(V)$. In other words, $\ell$-blades map only to $\ell$-blades under the induced action of $\psi$. The result then follows from the definition of grade reduction.

Corollary 5.8. If $\Phi$ represents blade conjugation on $V$, then $\Phi \rightarrow \varphi \searrow \Delta$ for some diagonal operator $\Delta$ on $V_{*}$. 
Remark 5.9. Using a suitably ordered basis, lowering operators are represented by lower triangular matrices, raising operators by upper triangular matrices, and grade preserving operators by block-diagonal matrices.

\subsection{Form-Induced Operators}

The quadratic form $Q$ naturally induces a linear operator $Q$ on $V$ defined so that the $Q$-inner product coincides with the Euclidean inner product. In other words,

$$
\langle\mathbf{x}, \mathbf{y}\rangle_{Q}=\langle Q(\mathbf{x}) \mid \mathbf{y}\rangle
$$

Definition 5.10. Given the operator $Q$ on $V$ induced by the quadratic form $Q$ on $V$, the linear operator $\mathfrak{Q}$ on $\mathcal{C} \ell_{Q}(V)$ induced by $Q$ is said to be form-induced by $Q$. In particular, given a canonical basis $\left\{\mathbf{e}_{1}, \ldots, \mathbf{e}_{n}\right\}$ for $V$, one defines $\mathfrak{Q}$ by

$$
\mathfrak{Q}\left(\mathbf{e}_{I}\right):=\sum_{J \in 2^{[n]}}\left(\prod_{\ell \in I \cap J} Q\left(\mathbf{e}_{\ell}\right)\right) \mathbf{e}_{J} .
$$

For convenience, write $Q \rightsquigarrow \mathfrak{Q}$ when (5.13) holds and $Q \rightarrow \mathfrak{Q}$.

As will be seen shortly, symmetric Kravchuk matrices are related to operators form-induced by negative definite quadratic forms. First, another well-known family of matrices is recalled.

The family $\mathfrak{H}$ of Sylvester-Hadamard matrices is defined as the collection of tensor (Kronecker) powers of the initial matrix $H=\left(\begin{array}{cc}1 & 1 \\ 1 & -1\end{array}\right)$. In particular, $\mathfrak{H}:=\left\{H^{\otimes n}: n \in \mathbb{N}\right\}$, where

$$
H^{\otimes n}=\underbrace{H \otimes H \otimes \cdots \otimes H}_{n \text { times }} .
$$

Remark 5.11. In terms of the Pauli matrices, $H=\sigma_{x}+\sigma_{z}$.

Writing $\{\bullet, \circ\}$ in place of $\{1,-1\}$, the matrices of $\mathfrak{H}$ for $n=2,3$ are:

$$
H^{\otimes 2}=\left(\begin{array}{cccc}
\bullet & \bullet & \bullet & \bullet \\
\bullet & 0 & \bullet & 0 \\
\bullet & \bullet & 0 & 0 \\
\bullet & 0 & 0 & \bullet
\end{array}\right), H^{\otimes 3}=\left(\begin{array}{cccccccc}
\bullet & \bullet & \bullet & \bullet & \bullet & \bullet & \bullet & \bullet \\
\bullet & 0 & \bullet & 0 & \bullet & 0 & \bullet & 0 \\
\bullet & \bullet & 0 & 0 & \bullet & \bullet & 0 & 0 \\
\bullet & 0 & 0 & \bullet & \bullet & 0 & 0 & \bullet \\
\bullet & \bullet & \bullet & \bullet & 0 & 0 & 0 & 0 \\
\bullet & 0 & \bullet & 0 & 0 & \bullet & 0 & \bullet \\
\bullet & \bullet & 0 & 0 & 0 & 0 & \bullet & \bullet \\
\bullet & 0 & 0 & \bullet & 0 & \bullet & \bullet & 0
\end{array}\right) .
$$

Obviously $H^{\otimes n}$ represents a linear operator on a $2^{n}$-dimensional vector space. Since the eigenvalues of the $n^{\text {th }}$ Hadamard matrix are $\pm 2^{n / 2}$, each with multiplicity $2^{n-1}$, it follows immediately that $H^{\otimes n}$ represents generalized conjugation by a blade of grade $2^{n-1}$. 
In the Clifford algebra of negative definite signature, that is, $\mathcal{C} \ell_{Q}(V) \cong \mathcal{C} \ell_{0, n}$, the Hadamard matrix represents the operator form-induced by $Q$. In particular, let $\mathfrak{H}$ denote the operator represented by $H^{\otimes n}$, with rows and columns indexed by integers $\left\{0, \ldots, 2^{n}-1\right\}$. Using the binary representation of integers, one sees that $\mathfrak{H}$ acts on basis blade $\mathbf{e}_{I}$ according to

$$
\mathbf{e}_{I} \mapsto \sum_{J \in 2^{[n]}}\left(\prod_{\ell \in I \cap J} Q\left(\mathbf{e}_{\ell}\right)\right) \mathbf{e}_{J}=\sum_{J \in 2^{[n]}}(-1)^{I \cap J} \mathbf{e}_{J},
$$

so that in terms of the Euclidean inner product,

$$
\left\langle\mathfrak{H}\left(\mathbf{e}_{I}\right) \mid \mathbf{e}_{J}\right\rangle=(-1)^{|I \cap J|}=\prod_{\ell \in I \cap J} Q\left(\mathbf{e}_{\ell}\right) .
$$

Relative to the $Q$-inner product, one finds

$$
\begin{aligned}
\left\langle\mathfrak{H}\left(\mathbf{e}_{I}\right), \mathbf{e}_{J}\right\rangle_{Q} & :=\left\langle(-1)^{|I \cap J|} \tilde{\mathbf{e}_{J}} \mathbf{e}_{J}\right\rangle_{0} \\
& =(-1)^{|I \cap J|+|J|} \\
& =(-1)^{|J \backslash I|}=\prod_{\{\ell \in J: \ell \notin I\}} Q\left(\mathbf{e}_{\ell}\right) .
\end{aligned}
$$

Regarding the symmetric Kravchuk matrix $\mathfrak{K}_{n}{ }^{\text {sym }}$ as a linear operator on $V_{*}$, the preceding discussion leads to the following theorem.

Lemma 5.12. Let $\mathcal{S}$ denote the operator on $V_{*}$ represented by the symmetric Kravchuk matrix $\mathfrak{K}_{n}{ }^{\mathrm{sym}}$. Let $\mathcal{H}$ denote the operator on $\mathcal{C} \ell_{Q}(V)$ represented by the $n^{\text {th }}$ Sylvester-Hadamard matrix, $H^{\otimes n}$, as described previously, and let $Q$ be negative definite on $V$. Then,

$$
Q \rightsquigarrow \mathcal{H} \searrow \mathcal{S} .
$$

Proof. It is known [11] that the $n^{\text {th }}$ symmetric Kravchuk matrix is obtained by summing entries of the $n^{\text {th }}$ Hadamard matrix by selecting rows and columns indexed by integers whose binary representations are of Hamming weights corresponding to the row and column index of the symmetric Kravchuk matrix. These Hamming weights correspond precisely to the grades of canonical blades in $\mathcal{C} \ell_{Q}(V)$, so that this summing is accomplished by grade deduction of the $n^{\text {th }}$ Hadamard matrix; i.e.,

$$
\left\langle\varepsilon_{i}|\mathcal{S}| \varepsilon_{j}\right\rangle=\sum_{\substack{\sharp \mathfrak{a}=i \\ \forall \mathfrak{b}=j}}\langle\mathfrak{a}|\mathcal{H}| \mathfrak{b}\rangle .
$$

Hence, $\mathcal{H} \searrow S$.

Along these lines, define the binomial grade operator $\Gamma$ on $\mathcal{C} \ell_{Q}(V)$ by

$$
\Gamma(\mathfrak{u}):=\left(\begin{array}{c}
|V| \\
\sharp \mathfrak{u}
\end{array}\right) \mathfrak{u} .
$$

The inverse is given by $\Gamma^{-1}(\mathfrak{u}):=\left(\begin{array}{c}|V| \\ \sharp \mathfrak{u}\end{array}\right)^{-1} \mathfrak{u}$. 
Remark 5.13. Let $\mathcal{H}^{\prime}$ be defined on $\mathcal{C} \ell_{Q}(V)$ by $\mathcal{H}^{\prime}:=\Gamma^{-1} \mathcal{H}$, and let $\mathfrak{x}$ denote a basis blade chosen randomly (with all blades having equal probability) from the canonical basis $\left\{\mathbf{e}_{I}: I \in 2^{[n]}\right\}$ of $\mathcal{C} \ell_{Q}(V)$, where $Q$ is negative definite. The operator $\mathcal{H}^{\prime}$ then has the following probabilistic interpretation:

$$
\left\langle\mathcal{H}^{\prime}\left(\mathbf{e}_{I}\right), \mathbf{e}_{J}\right\rangle_{Q}=(-1)^{|I \cap J|} \mathbb{P}\left(\mathfrak{x}=\mathbf{e}_{I}|\sharp \mathfrak{x}=| I \mid\right) .
$$

Lemma 5.14. Regarding the $n^{\text {th }}$ Kravchuk matrix $\mathfrak{K}_{n}$ as an operator on $V_{*}$, let $\mathcal{H}^{\prime}$ be defined on $\mathcal{C} \ell_{Q}(V)$ by $\mathcal{H}^{\prime}:=\Gamma^{-1} \mathcal{H}$. Then,

$$
\mathcal{H}^{\prime} \searrow \mathfrak{K}_{n} .
$$

Proof. In terms of canonical basis blades,

$$
\mathcal{H}^{\prime}\left(\mathbf{e}_{I}\right)=\sum_{J \in 2^{[n]}}(-1)^{|I \cap J|}\left(\begin{array}{c}
n \\
|J|
\end{array}\right)^{-1} \mathbf{e}_{J} .
$$

Denote the grade-reduction of $\mathcal{H}^{\prime}$ by $X$. Then,

$$
\begin{aligned}
\left\langle\varepsilon_{i}|X| \varepsilon_{j}\right\rangle & =\sum_{|I|=i,|J|=j}(-1)^{|I \cap J|}\left(\begin{array}{c}
n \\
|J|
\end{array}\right)^{-1} \\
& =\left(\begin{array}{c}
n \\
|J|
\end{array}\right)^{-1}\left\langle\varepsilon_{i}\left|\mathfrak{K}_{n}{ }^{\mathrm{sym}}\right| \varepsilon_{j}\right\rangle \\
& =\left\langle\varepsilon_{i}\left|\mathfrak{K}_{n}\right| \varepsilon_{j}\right\rangle .
\end{aligned}
$$

\section{Conclusion}

While the full scope of the relationship between Kravchuk polynomials and Clifford algebras lies beyond the current work, a few parting comments are in order.

Spectral properties of Clifford conjugation operators are of interest in quantum probability. In quantum probability, self-adjoint operators are analogous to random variables, with the expectation being given by the operator's trace. As seen in Lemma 2.3, $\Phi_{\mathfrak{u}}$ is a $Q$-orthogonal transformation on $V$. An immediate consequence is that $\Phi_{\mathfrak{u}}$ is self-adjoint with respect to the $Q$-inner product and that $\Phi_{\mathfrak{u}}$ is correctly regarded as a quantum random variable. From the spectral properties uncovered in Theorem 2.2, one now interprets $\Phi_{\mathfrak{u}}$ as a quantum random variable taking values \pm 1 .

From a practical viewpoint, blade conjugation operators play a role in computing Clifford factorizations of blades, which can then be used in the construction of Appell systems [22, 25]. Moreover, "nearest blade" approximations can be useful for efficient symbolic computation. Clifford factorizations of blades can be obtained from eigenspace bases of conjugation operators. In terms of a 
fixed basis for the vector space $V$, the expansion of an arbitrary blade $\mathfrak{u}$ can contain up to $\left(\begin{array}{c}n \\ \mathfrak{t u}\end{array}\right)$ nonzero coefficients. On the other hand, the $\sharp \mathfrak{u}$ factors of $\mathfrak{u}$ are vectors in the $n$-dimensional space $V$; thus, only $n \sharp \mathfrak{u}$ scalars are needed to determine $\mathfrak{u}$.

Finally, for readers interested in practical applications like image processing [2, 28] or error-correcting codes [6, 19], the Kravchuk transform [13] extends easily from $V$ to the Clifford algebra $\mathcal{C} \ell_{Q}(V)$ via a blade conjugation operator. A detailed treatment of "Clifford-Kravchuk" transforms is to be a subject of future work.

Because the dimension of the Clifford algebra is exponential in the dimension of its generating vector space, scalability makes computations for practical applications challenging. However, Clifford algebra computations are generally simplified by symbolic treatment. Avoiding explicit matrix representations of the algebras tends to make computations both faster and more intuitive. A number of software packages exist for performing symbolic computations with Clifford algebras, including the author's CliffMath package for Mathematica, available at http://www.siue.edu/ sstaple/index_files/research.htm.

\section{Acknowledgment}

The author thanks Philip Feinsilver for a number of thought-provoking discussions over the years.

\section{References}

[1] N.M. Atakishiyev, K.B. Wolf, Fractional Fourier-Kravchuk transform, J. Opt. Soc. Amer. A, 147 (1997), 1467-1477.

[2] T. Batard, M. Berthier, C. Saint-Jean, Clifford-Fourier Transform for Color Image Processing, in Geometric Algebra Computing, E. Bayro-Corrochano, G. Scheuermann, Eds., Springer London, pp. 135-162, 2010. http://dx. doi.org/10.1007/978-1-84996-108-0_8.

[3] F.A. Berezin, Introduction to Superanalysis, D. Reidel Publishing Co., Dordrecht, 1987.

[4] P. Delsarte, Bounds for restricted codes, by linear programming, Philips Res. Reports, 27 (1972), 272-289.

[5] P. Delsarte, Four fundamental parameters of a code and their combinatorial significance, Info. \& Control, 23 (1973), 407-438.

[6] P. Delsarte, An algebraic approach to the association schemes of coding theory, Philips Research Reports Supplements, No. 10, N.V. Philips' Gloeilampenfabrieken, Eindhoven, Netherlands, 1973.

[7] C.F. Dunkl, A Krawtchouk polynomial addition theorem and wreath products of symmetric groups, Indiana Univ. Math. J., 25 (1976), 335-358. 
[8] C.F. Dunkl, D.F. Ramirez, Krawtchouk polynomials and the symmetrization of hypergraphs, SIAM J. Math. Anal., 5 (1974), 351-366.

[9] P. Feinsilver, R. Fitzgerald, The spectrum of symmetric Kravchuk matrices, Linear Algebra and Its Applications, 235 (1996), 121-139.

[10] P. Feinsilver, U. Franz, R. Schott, Duality and multiplicative processes on quantum groups, J. Th. Prob., 10 (1997), 795-818.

[11] P. Feinsilver, J. Kocik, Krawtchouk polynomials and Krawtchouk matrices, Recent Advances in Applied Probability, R. Baeza-Yates, J. Glaz, H. Gzyl, J. Hüsler, J.L. Palacios, (Eds.), Springer, 2005, pp. 115-141.

[12] P. Feinsilver, J. Kocik, Krawtchouk matrices from classical and quantum random walks, Contemporary Mathematics, 287 (2001), 83-96.

[13] P. Feinsilver, R. Schott, On Krawtchouk transforms, Intelligent Computer Mathematics, Proc. 10th Intl. Conf. AISC 2010, LNAI 6167, 6475.

[14] P. Feinsilver, R. Schott, Krawtchouk polynomials and finite probability theory, In: H. Heyer (ed.) Probability Measures on Groups X (Olberwolfach 1990), pp. 129-135, Plenum, New York, 1991.

[15] S. Gudder, Quantum Probability, Academic Press, Boston, 1988.

[16] T. Koornwinder, Krawtchouk polynomials, a unification of two different group theoretic interpretations, SIAM J. Math. Anal., 13 (1982), 1011-1023.

[17] V.I. Levenstein, Krawtchouk polynomials and universal bounds for codes and design in Hamming spaces, IEEE Transactions on Information Theory, 41 (1995), 1303-1321.

[18] P. Lounesto, E. Latvamaa, Conformal Transformations and Clifford Algebras, Proc. Am. Math. Soc., 79 (1980), 533-538.

[19] F. J. MacWilliams, N. J. A. Sloane, Theory of Error-Correcting Codes, North-Holland, 1977.

[20] P.A. Meyer, Quantum Probability for Probabilists, Lecture Notes in Mathematics 1538, Springer-Verlag, Berlin,1995.

[21] K.R. Parthasarathy, An Introduction to Quantum Stochastic Calculus, Birkhäuser Verlag, Basel, 1992.

[22] R. Schott, G.S. Staples, On the role of blade factorization in constructing Clifford Appell systems, Prépublications de l'Institut Élie Cartan No. 2011/024, 2011.

[23] R. Schott, G.S. Staples, Operator calculus and Appell systems on Clifford algebras, Int. J. of Pure and Appl. Math., 31 (2006), 427-446. 
[24] R. Schott, G.S. Staples. Operator homology and cohomology in Clifford algebras, Cubo, A Mathematical Journal, 12 (2010), 299-326.

[25] R. Schott, G.S. Staples, Operator calculus and invertible Clifford Appell systems: theory and application to the n-particle fermion algebra, Infinite Dimensional Analysis, Quantum Probability, and Related TopicsInfin. Dimens. Anal. Quantum. Probab. Relat. Top. 16, 1350007 (2013) [23 pages] DOI: $10.1142 / \mathrm{S} 0219025713500070$.

[26] D. Shale, W.F. Stinespring, States of the Clifford algebra, Annals of Math, 80 (1964), 365-381.

[27] G. Szëgo, Orthogonal Polynomials, Amer. Math. Soc., Providence, 1955.

[28] P-T. Yap, R. Paramesran, Image analysis by Krawtchouk moments, IEEE Transactions on image processing, 12 (2003), 1367-1377. 